This item was submitted to Loughborough's Research Repository by the author.

Items in Figshare are protected by copyright, with all rights reserved, unless otherwise indicated.

\title{
Multi-classifier for reinforced concrete bridge defects
}

PLEASE CITE THE PUBLISHED VERSION

https://doi.org/10.1016/j.autcon.2019.04.019

PUBLISHER

(c) Elsevier

VERSION

AM (Accepted Manuscript)

PUBLISHER STATEMENT

This paper was accepted for publication in the journal Automation in Construction and the definitive published version is available at https://doi.org/10.1016/j.autcon.2019.04.019.

\section{LICENCE}

CC BY-NC-ND 4.0

\section{REPOSITORY RECORD}

Huthwohl, Philipp, Ruodan Lu, and loannis Brilakis. 2019. "Multi-classifier for Reinforced Concrete Bridge Defects". Loughborough University. https://hdl.handle.net/2134/37724. 
3

$4 \quad{ }^{1}$ Department of Engineering, University of Cambridge, United Kingdom

$5 \quad{ }^{2 *}$ Corresponding author, School of Architecture, Building and Civil Engineering, Loughborough

6 University, United Kingdom, and Darwin College, University of Cambridge, E-mail: r.lu@,lboro.ac.uk,

7 r1508@cam.ac.uk

Philipp Hüthwohl ${ }^{1}$, Ruodan Lu$^{2 *}$ A.M. ASCE; Ioannis Brilakis ${ }^{3}$, M. ASCE

9 Abstract

Classifying concrete defects during a bridge inspection remains a subjective and laborious task. The risk of getting a false result is approximately $50 \%$ if different inspectors assess the same concrete defect. This is significant in the light of an over-aging bridge stock, decreasing infrastructure maintenance budgets and catastrophic bridge collapses as happened in 2018 in Genoa, Italy. To support an automated inspection and an objective bridge defect classification, we propose a three-staged concrete defect classifier that can multiclassify potentially unhealthy bridge areas into their specific defect type in conformity with existing bridge inspection guidelines. Three separate deep neural pre-trained networks are fine-tuned based on a multisource dataset consisting of self-collected image samples plus several Departments of Transportation inspection databases. We show that this approach can reliably classify multiple defect types with an average mean score of $85 \%$. Our presented multi-classifier is a contribution towards developing a mostly or fully inspection schema for a more cost-effective and more objective bridge inspection.

Key words: Concrete defect classification, automated bridge inspection, defect detection, crack detection, spalling detection, scaling detection, efflorescence detection, rust staining detection, exposed reinforcement detection 


\section{Introduction}

26 Bridges are vital assets and critical structures in road networks. Their weight restrictions and closures due 27 to deterioration have drastic implications on a country's economic success and the road users' satisfaction $28[1,2]$. Human bridge inspectors monitor bridge condition based on a typically biannual manual bridge 29 inspection scheme [3].

30 Previous research yielded methods that can automatically generate fully textured as-is geometrical digital bridge twins (Figure 1) [4, 5]. By analysing the texture of such models, the search space for inspection can be reduced over $90 \%$ to only potentially unhealthy areas [5]. However, answering the question of which type of defect is an unsolved problem. Currently, this question is answered manually by a human inspector. Existing evidences suggest that about $50 \%$ of the given condition ratings are incorrect or differ between inspectors [6].
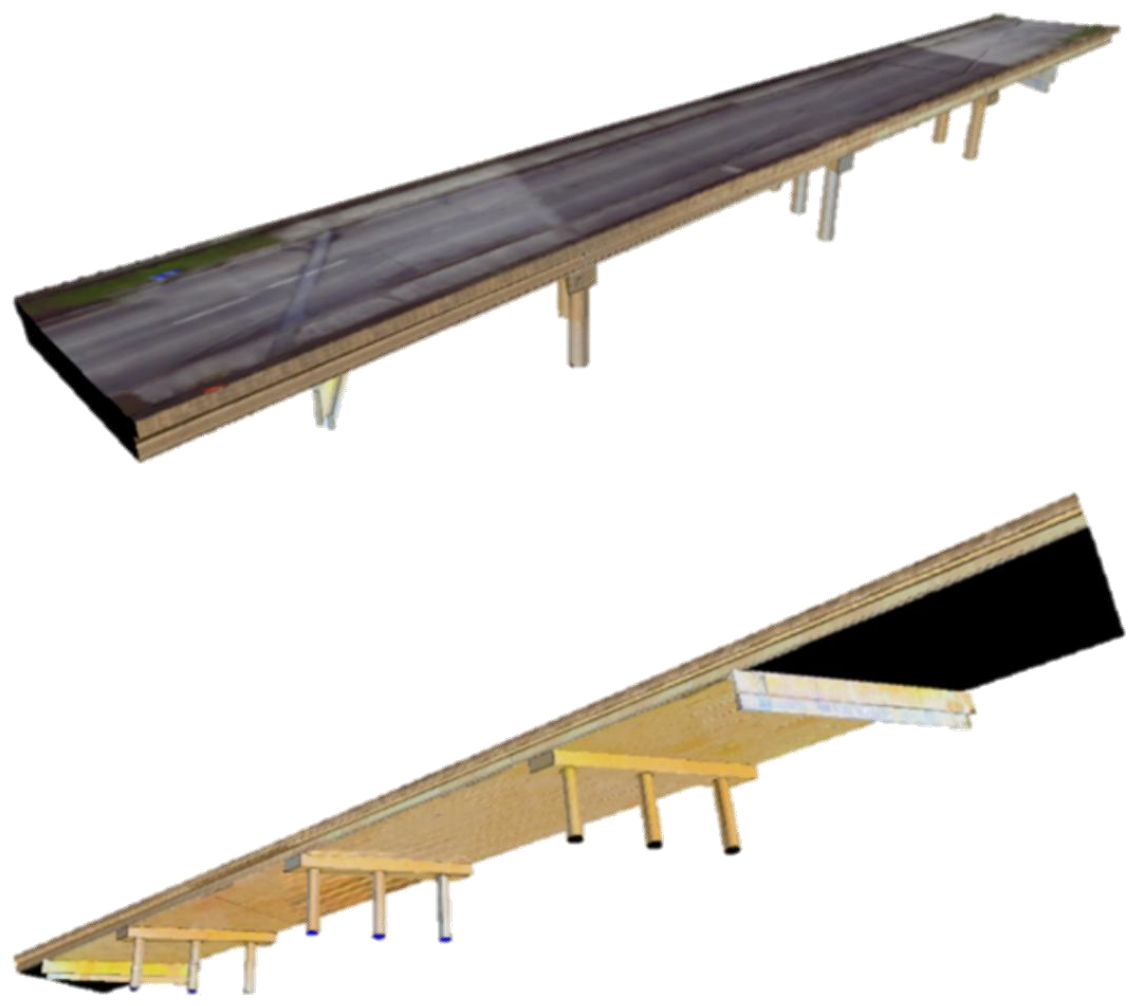

The significance of this problem relates to the fact that approximately 720,000 bridges are inspected 
bridges and cost structure in the US and EU [7, 8]. Ongoing deterioration of the bridge stock leads to a more rigorous inspection plan for critical bridges. Hence, there is an even increasing number of bridge inspections per year [9]. Furthermore, $£ 18$ billion are spent based on the bridge condition information in the US and EU yearly in order to maintain the bridge stock and to prevent a further decline in the overall bridge infrastructure condition $[10,11]$. An automated defect classification method is therefore urgently needed and can contribute to a more objective and repeatable inspection decision as well as a reduction of workload and inspection costs.

The process of bridge inspection, and especially the defect classification, is defined by bridge inspection guidelines which are published by respective authorities (e.g. [3, 12-15]). A single inspector or a team of inspectors should list all defects identified during a bridge inspection and manually enter these results in a bridge inspection report. AEC industry has developed commercial tools to support inspectors during inspections. For example, Leica Geosystems AG builds a total station with integrated crack width gauge to measure and document crack properties from a remote position. An inspector aims at a crack position and then determines the crack width by manually comparing the actual crack width with a list of reference crack widths which are overlaid in the field of view of inspectors [16]. Nivo-i, a total station from a Nikon Trimble joint venture, can automatically detect, localize, and measure cracks down to a width of $0.2 \mathrm{~mm}$ from a distance of $30 \mathrm{~m} \mathrm{[17].} \mathrm{Crack} \mathrm{coordinates} \mathrm{and} \mathrm{widths} \mathrm{are} \mathrm{documented} \mathrm{in} \mathrm{images} \mathrm{or} \mathrm{CAD} \mathrm{files.} \mathrm{However,}$ cracks are the only documented defect types, both total stations do not consider other ones.

The next chapter presents an in-depth analysis of what methods have been proposed in previous research to overcome the above-mentioned limitations followed by identifying gaps in knowledge and the objective of this paper. Section 3 describes the newly developed hierarchical classification methodology, aiming to solve the multi-classification problem. This is assessed and discussed qualitatively and quantitatively in Section 4. Finally, Section 5 presents the scientific contribution and conclusions of this paper.

\section{$63 \quad 2$ Background}

\subsection{Defect Classification Requirements}

Bridge inspection guidelines and the defect classification requirements serve as reference bar for an objective evaluation of the state-of-research regarding the problem of defect classification. Five inspection 
defect classes are spalling, cracks, rust staining, efflorescence, scaling, abrasion/wear, exposed reinforcement and general (which combines defects that are specific to one inspection guideline). Abrasion and wear are difficult to detect visually, and different definitions exist [19]. However, from analysing these inspection guidelines regarding the classification specifications reveals that solely detecting the defect type is not enough to meet the guideline requirements. We therefore identified three additional constraints which are considered as reference bar for evaluating classification methods for bridge inspection defects for the scope of this work: (1) multiple defects, (2) defect combinations, and (3) scale independence.

The first requirement is the ability to detect multiple defects types. Each of the guidelines lists several types of defects. Therefore, a defect classifier must be able to reliably detect multiple types of defects simultaneously.

Then, an analysis of the inspection guidelines discovered that some defects can appear as a combination whereas other defect types are mutually exclusive. For example, spalls and cracks can occur in the same location as shown in Figure 2. However, looking into Table 1 taken from an inspection manual, it is either cracks or spalls which has to be identified, not both. On the other hand, spalls and exposed reinforcement can very well appear together according to the inspection manual. A defect classifier should simultaneously detect multiple defect classes while considering others are mutually exclusive.

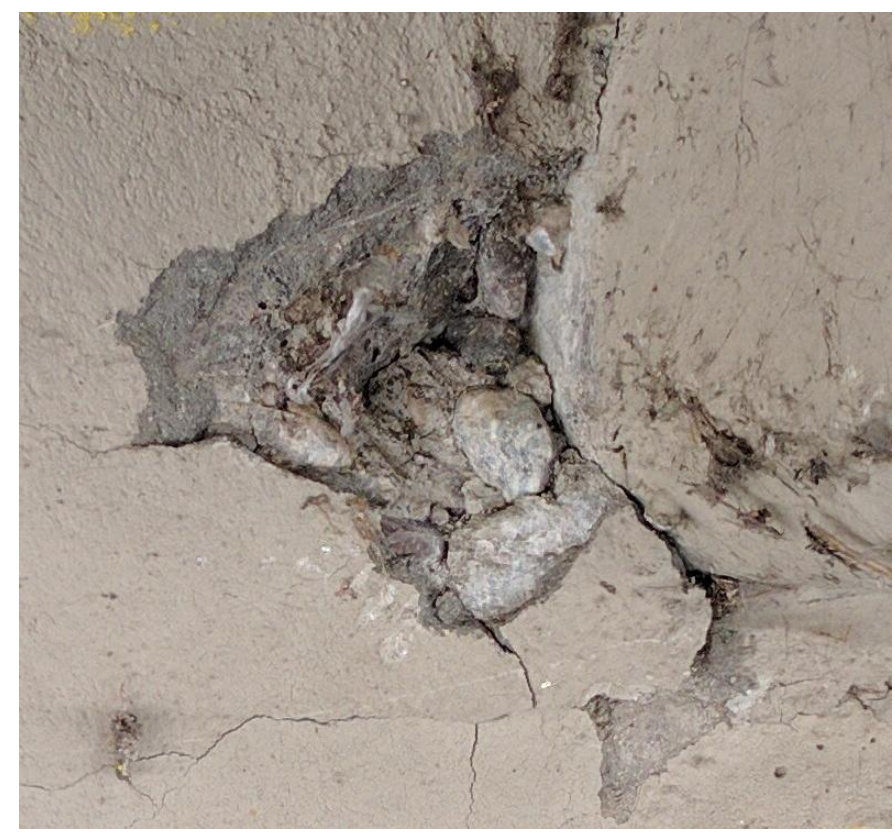




\begin{tabular}{|c|c|c|c|c|c|}
\hline & 1 & 2 & 3 & 4 & 5 \\
\hline 0.1 & No spalls & $\begin{array}{l}\text { Minor localised } \\
\text { spalls exposing } \\
\text { shear links }\end{array}$ & $\begin{array}{l}\text { Major localised } \\
\text { spalls exposing } \\
\text { shear links } \\
\text { and main bars } \\
\text { with general } \\
\text { corrosion }\end{array}$ & $\begin{array}{l}\text { Joined up deep } \\
\text { spalls exposing } \\
\text { shear links and } \\
\text { main bars with } \\
\text { general and } \\
\text { pitting } \\
\text { corrosion }\end{array}$ & Collapsed \\
\hline 0.2 & $\begin{array}{l}\text { Hairline cracks, } \\
\text { Difficult to } \\
\text { detect visually }\end{array}$ & $\begin{array}{l}\text { Cracks and } \\
\text { crazing in areas } \\
\text { of low flexural } \\
\text { behaviour } \\
\text { (cracks less } \\
\text { than } 0.3 \mathrm{~mm} \text { ) }\end{array}$ & $\begin{array}{l}\text { Cracks and } \\
\text { crazing in areas } \\
\text { of high flexure. } \\
\text { Cracks approx. } \\
1 \mathrm{~mm} \text { and } \\
\text { easily visible) }\end{array}$ & $\begin{array}{l}\text { Wide/deep } \\
\text { cracks (more } \\
\text { than } 2 \text { mm). } \\
\text { Shear cracks }\end{array}$ & $\begin{array}{l}\text { Element unable } \\
\text { to function due } \\
\text { to structural } \\
\text { cracks }\end{array}$ \\
\hline 0.3 & $\begin{array}{l}\text { No signs of } \\
\text { damage to } \\
\text { prestressing }\end{array}$ & $\begin{array}{l}\text { Substandard } \\
\text { grouting of } \\
\text { ducts (may not } \\
\text { be visible) }\end{array}$ & $\begin{array}{l}\text { Cracks along } \\
\text { line of } \\
\text { prestressing } \\
\text { duct }\end{array}$ & $\begin{array}{l}\text { Exposed } \\
\text { prestressing } \\
\text { cables }\end{array}$ & $\begin{array}{l}\text { Failed } \\
\text { prestressing } \\
\text { cables }\end{array}$ \\
\hline 0.4 & $\begin{array}{l}\text { No signs of } \\
\text { delamination }\end{array}$ & $\begin{array}{l}\text { Early signs of } \\
\text { delamination } \\
\text { e.g. cracks with } \\
\text { rust staining }\end{array}$ & $\begin{array}{l}\text { Delamination } \\
\text { in areas of low } \\
\text { flexural and/or } \\
\text { shear action }\end{array}$ & $\begin{array}{l}\text { Delamination } \\
\text { in areas of high } \\
\text { flexural and/or } \\
\text { shear action }\end{array}$ & $\begin{array}{l}\text { Failure due to } \\
\text { delaminated } \\
\text { bars }\end{array}$ \\
\hline 0.5 & $\begin{array}{l}\text { No signs of } \\
\text { thaumasite or } \\
\text { freeze-thaw } \\
\text { attack }\end{array}$ & $\begin{array}{l}\text { Slight cracking } \\
\text { caused by } \\
\text { thaumasite or } \\
\text { freeze-thaw }\end{array}$ & $\begin{array}{l}\text { Moderate } \\
\text { thaumasite or } \\
\text { freeze-thaw } \\
\text { attack }\end{array}$ & $\begin{array}{l}\text { Major } \\
\text { thaumasite } \\
\text { or freezethaw } \\
\text { attack }\end{array}$ & $\begin{array}{l}\text { Failure due to } \\
\text { thaumasite or } \\
\text { freeze-thaw } \\
\text { attack }\end{array}$ \\
\hline
\end{tabular}

Note: Each element is assessed by its dominant defect. The column number in combination with the row number gives an element condition. E.g. a condition rating of 3.2 describes cracks in a high flexure area.

The third requirement of a bridge inspection defect classifier is the ability to classify defects independently from the defect scale. Different defect types appear at different scales. Some defects, such as spalling or scaling, affect a considerable, typically two-dimensional area in a surface texture. Dissecting this area into chunks impedes the correct classification. For example, taking an inner part of a high-resolution spalling defect could appear as healthy concrete as depicted in Figure 3. A correct classification should include the complete affected area. In contrast, rescaling the affected area to a different resolution does still allow for correct defect classification for this defect type. On the other hand, cracks are represented as onedimensional formation in the surface texture. Specifically, thin cracks may possibly be present within only a small number of pixels but bear a potential structural risk to a bridge. Rescaling an image with a crack could completely remove the defect from the surface texture and makes it impossible to detect even for a human inspector. Dissecting, however, does not change the characteristics of defects. In other words, a crack remains a crack even when inspecting only a small section of a wider crack. A defect classifier has to be able to detect defects invariantly from the scale. 

for defects detection in bridge inspection.

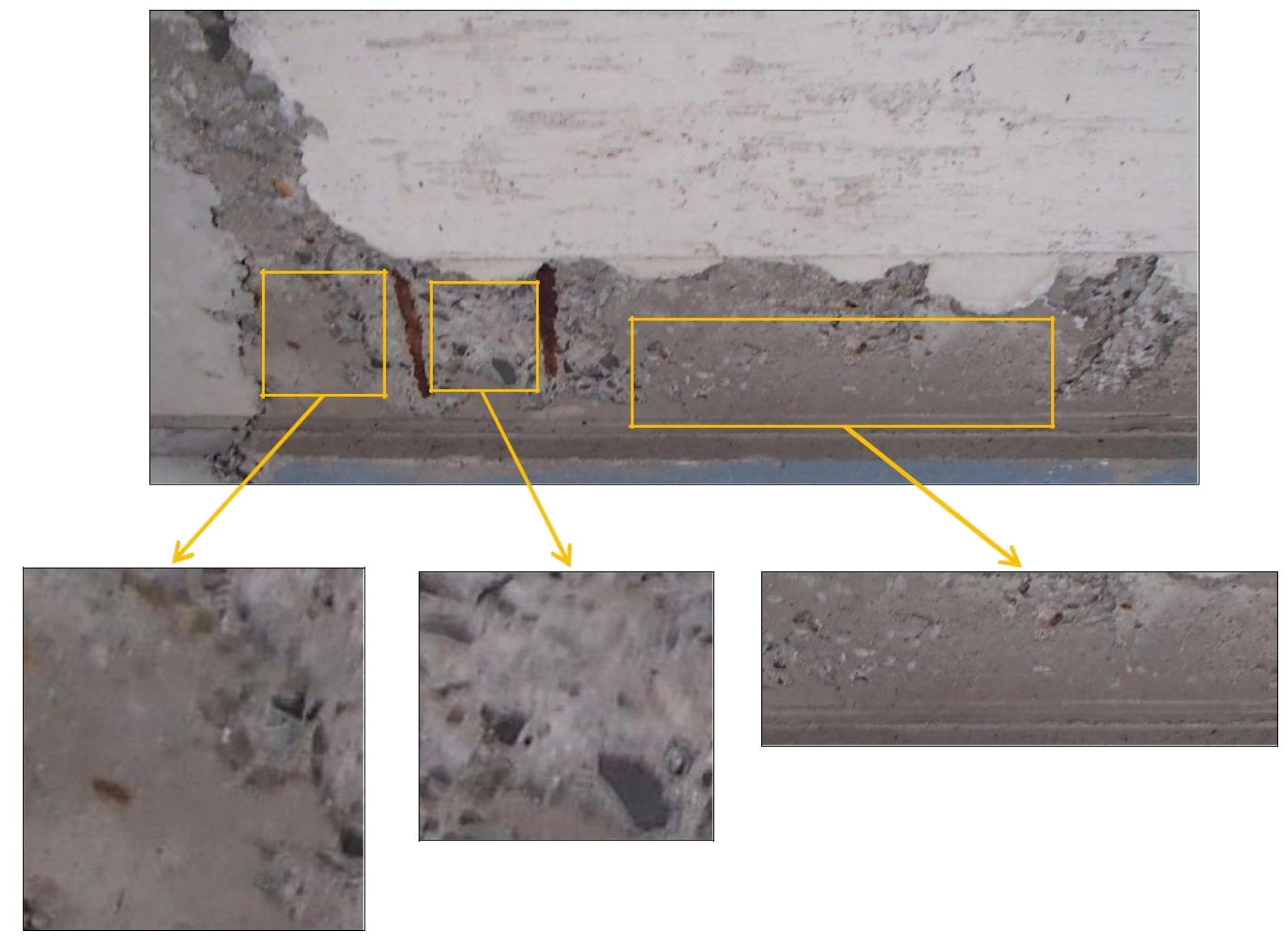

Figure 3 Example of patch extraction from spalling

\subsection{Relevant Studies}

Researchers have addressed the problem of detecting concrete defects in several studies. There is no consistent use of the term detection and classification, a universal definition does not exist. Figure 4 describes the six steps of condition assessment. In this work, we define "Classification" (Step 3) as the process of assigning one or multiple labels to an image based on image content. It does not include the extraction of the exact pixels affected by the defect. "Unhealthy Detection" (Step 2), in contrast, is the prepending process which extracts potentially unhealthy surface patches out of the complete surface texture [20]. "Texture Mapping" (Step 1) and "Unhealthy Detection" (Step 2) were addressed in the authors' previous works $[5,18]$. Subsequent steps, which are beyond the scope of this work, could be the semantic segmentation, the property extraction, and the condition rating. "Semantic Segmentation" (Step 4) indicates the location of a defect at a pixel level and would include a definition of which parts of a defect are actually 
part of a defect. Then, "Property Extraction" (Step 5) measures the defect type dependent properties such as width and orientation for a crack. Finally, "Condition Rating" (Step 6) completes the assessment by assigning a condition rating.

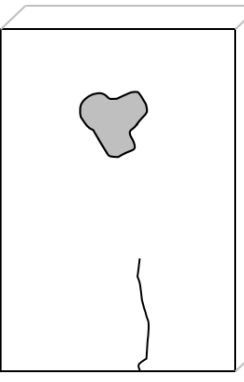

Texture Mapping



Unhealthy Detection



Classification

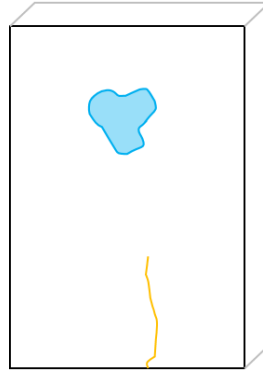

Semantic Segmentation

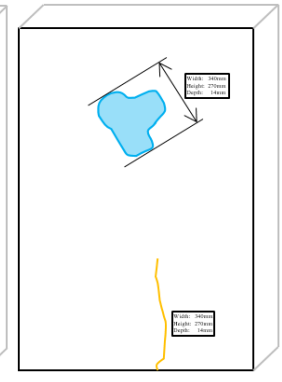

Property Extraction

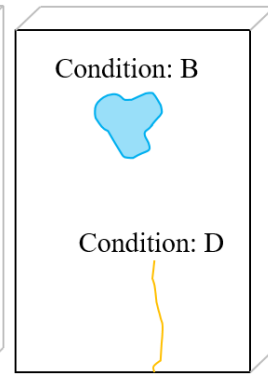

Condition Rating

Figure 4 Steps of classification toward condition assessment

Much of the work on defect classification has concentrated on detecting a single defect class (actually two classes: defect and background), i.e. predominant cracks. Koch et al. [21] did a thorough review on these methods. Methods of detecting cracks follow a traditional computer vision approach of a pre-processing step to enhance contrast and reduce noise (median filter [22], morphologic operator [23], background subtraction [24]), followed by a feature extraction step (Canny [25], linear discriminant analysis of multiple features [26], Otsu [27]), and finally a classification step (support vector machine [28], AdaBoost [29], neural network [30]). Prasanna et al. [29] achieved an accuracy of 90\% on thousands of tests cracks. Detecting defects other than cracks has been less profoundly studied. German et al. [31] used a combination of thresholding and a template matching method to detect and measure column spalling in a post-earthquake scenario. Their work included the detection of horizontally and vertically exposed reinforced concrete and they achieved an average precision of $81.1 \%$ and a sensitivity of $80.2 \%$ at a pixel level. Existing single defect class detectors identify a specific defect type, for example a crack, at a pixel level in a rather small image where the defect is shown in a considerable part or not at all. However, existing classifiers do not investigate instances where a defect fills a vanishingly small number of pixels. They are also validated based on a pixel level rather than an instance level. This restriction can lead to misleading validation results.

It is worth noting that, before extracting the exact shape of a defect at a pixel level, one needs to determine which kind of a defect is of interest. Defect proximity might provide helpful context information for correct classification and for distinguishing from other defect types. It is, for example, in case of a crack that humidity enters the material and turns the direct crack environment dark. In addition, a single class detector focuses on detecting a specific class only. Using it without the knowledge of the expected defect type will 
lead to a confusion, i.e. either assign multiple labels for the same pixel, or chaotic defect class assignments per pixel which then have to be consolidated.

Feng et al. [32] proposed a method to detect different defect types using a deep neural network. They trained four separate classifiers, one for each of the defect class cracks, deposit, water leakage, and one for the combination of the former three. Their method achieved a precision of $79.5 \%$ and a sensitivity of $65.0 \%$. This method does show that deep neural networks can distinguish concrete defect types. However, it cannot exclusively assign defect labels as the defect type-dependent classifiers are trained separately and no method is presented on how to combine the classification results.

\subsection{Gaps in knowledge \& Objective}

Although many different defect classification/detection methods exist, there is no method that can identify multiple defect types by simultaneously considering the system of mutually exclusive defect combinations and sequentially cascading defect types as required by the inspection guidelines. Therefore, the objective of this paper is to develop a multi-classifier that can assign none, one, or multiple defect labels to an image containing potentially unhealthy areas. The possible defect combinations do not depend on the method itself but are dictated by the underlying inspection guideline.

\section{Methodology}

We assume the detection of the unhealthy surface patches from the complete surface texture is properly done, meaning that the most useful defect images have been selected. Then, we propose a three-stage multiclassifier to solve the previously mentioned challenges of defect classification. The novelty of our multiclassifier lies in the fact that it follows a three-staged hierarchical structure to analyse the expected defect combinations and types from the inspection guidelines. Specifically, the scope of this work is described in Table 2. We list eight different classes: spalling, cracks, rust staining, efflorescence, scaling, abrasion/wear, exposed reinforcement, and general defects. Table 2 shows that not all classes can occur exclusively. The ones that can occur exclusively are spalling, cracks, efflorescence, scaling, abrasion/wear, and general defects. Abrasion/wear is excluded because it is not a texture defect which is visually detectable but one that alters the three-dimensional shape. Exposed reinforcement occurs in combination with spalling, scaling, and efflorescence. Rust staining occurs with exposed reinforcement. We describe the resulting defect hierarchy in Figure 5. This hierarchy can now be used to implement the defect detection in three 
171 stages: the first stage is a multi-classifier with five classes plus a complementary "no defect" class; the 172 second stage is a binary classifier to decide on the presence of exposed reinforcement; and the third stage 173 is also a binary classifier to decide on the presence of rust staining. Stage two, the detection of the exposed 174 reinforcement, is only called in case the first stage classifies the defect to be relevant for exposed 175 reinforcement and accordingly the third stage is only called if the exposed reinforcement is detected. The 176 classification happens for each potentially unhealthy surface patch. Each surface patch can have multiple 177 defects. The possible classification results are thus:

$178 \quad-\quad$ Spalling

$179 \quad-\quad$ Spalling with exposed reinforcement

$180-$ Cracks

181 - Efflorescence

$182 \quad-\quad$ Efflorescence with exposed reinforcement

183 - Efflorescence with exposed reinforcement and rust staining

$184 \quad-$ Scaling

185 - Scaling with exposed reinforcement

186 - Scaling with exposed reinforcement and rust staining

187 - General defect

188 - No defect

189

190

191

192

193

194

195

196

197

198

199

200

201

202

203

204

205

206 


\begin{tabular}{|c|c|}
\hline Surface defect & Property \\
\hline $\begin{array}{l}\text { Spalling / } \\
\text { Exposed Rebar / } \\
\text { Corrosion }\end{array}$ & $\begin{array}{l}\text { Diameter } \\
\text { Depth } \\
\text { Shear link rebar visible } \\
\text { Main rebar visible } \\
\text { General corrosion on rebar } \\
\text { Pitting corrosion on rebar }\end{array}$ \\
\hline Cracks & $\begin{array}{l}\text { Width } \\
\text { Dropping down } \\
\text { Going up } \\
\text { Orientation in relation to the support } \\
\text { Area of high flexural behaviour } \\
\text { Area of high shear behaviour in area } \\
\text { Close to support }\end{array}$ \\
\hline Delamination & $\begin{array}{l}\text { Cracks } \\
\text { Rust staining } \\
\text { Area of high flexural behaviour } \\
\text { Area of high shear behaviour in area }\end{array}$ \\
\hline Freeze-thaw & $\begin{array}{l}\text { Accompanied by other defects } \\
\text { Other defects structurally relevant }\end{array}$ \\
\hline Efflorescence & $\begin{array}{l}\text { Severity } \\
\text { Peeling surface } \\
\text { Exposed Reinforcement }\end{array}$ \\
\hline Scaling & $\begin{array}{l}\text { Depth } \\
\text { Coarse aggregate exposed } \\
\text { Exposed Reinforcement }\end{array}$ \\
\hline Abrasion / Wear & $\begin{array}{l}\text { Coarse aggregate exposed } \\
\text { Exposed Reinforcement }\end{array}$ \\
\hline
\end{tabular}




After defining classes and a classification hierarchy, it is now possible to determine how decisions are

212 made. Each of the three classifiers outputs one set of class scores for one input instance. It is decided

213 whether the next stage is executed depending on the result of each stage. This decision-making process is

214 depicted in Figure 6. In general, for each classification stage the class label which achieved the highest

215 score is selected following the assumption that the classification result is correct.

216 It is hypothesized that the presented classifier hierarchy in combination with a state-of-the art image 217 classifier can reliably detect multiple defect-types simultaneously and none, one or, multiple classification

218 labels can be assigned. The hypothesis is tested by classifying a set of images which is unknown to the 219 classifier. The robustness of the classifier is measured using classification quality measures. The results are 220 presented in a confusion matrix. The quality measures are based on the numbers of possible classification 221 outcomes. In the case of a binary classification, there are four groups of classification results: number of 222 positive samples that are classified as positive (true positive, i.e. TP), number of negative samples that are 223 classified as negative (true negative, i.e. TN), number of positive samples that are classified as negative 224 (false negative, i.e. FN), and number of negative samples that are classified as positive (false positive, i.e. 225 FP). For the case of a non-binary classifier, all samples belonging to a corresponding group are summed 226 up. Quality measures can then be defined using these four groups of labels.

227 Formula ( 1 defines sensitivity, the fraction of getting a positive classification result for a positive sample.

$$
\text { Sensitivity }=\frac{T P}{T P+T N}
$$

228 Specificity in Formula (2 is the equivalent to sensitivity for negative samples, which is also called true 229 negative rate.

$$
\text { Specificity }=\frac{T N}{T N+F P}
$$

230 Precision in Formula (3 is the fraction of samples classified as positive that are actually positive. Negative 231 predictive value is the equivalent for negative samples. The formula for this is not listed separately.

$$
\text { Precision }=\frac{T P}{T P+F P}
$$

232 Accuracy in Formula (4 is the fraction of a sample being classified correctly, independently from its class.

$$
\text { Accuracy }=\frac{T P+T N}{T P+F P+T N+F N}
$$


233 In general, accuracy is a reliable measurement for assessing the overall performance of a classifier.

234 However, it is biased towards the predominant class if classes are unbalanced. For this reason, the $F_{1}$ score 235 is introduced which takes both false positives and false negatives into account. It is the harmonic mean of 236 precision and specificity as defined in Formula 5.

$$
F_{1}=2 * \frac{\text { precision } * \text { sensitivity }}{\text { precision }+ \text { sensitivity }}
$$

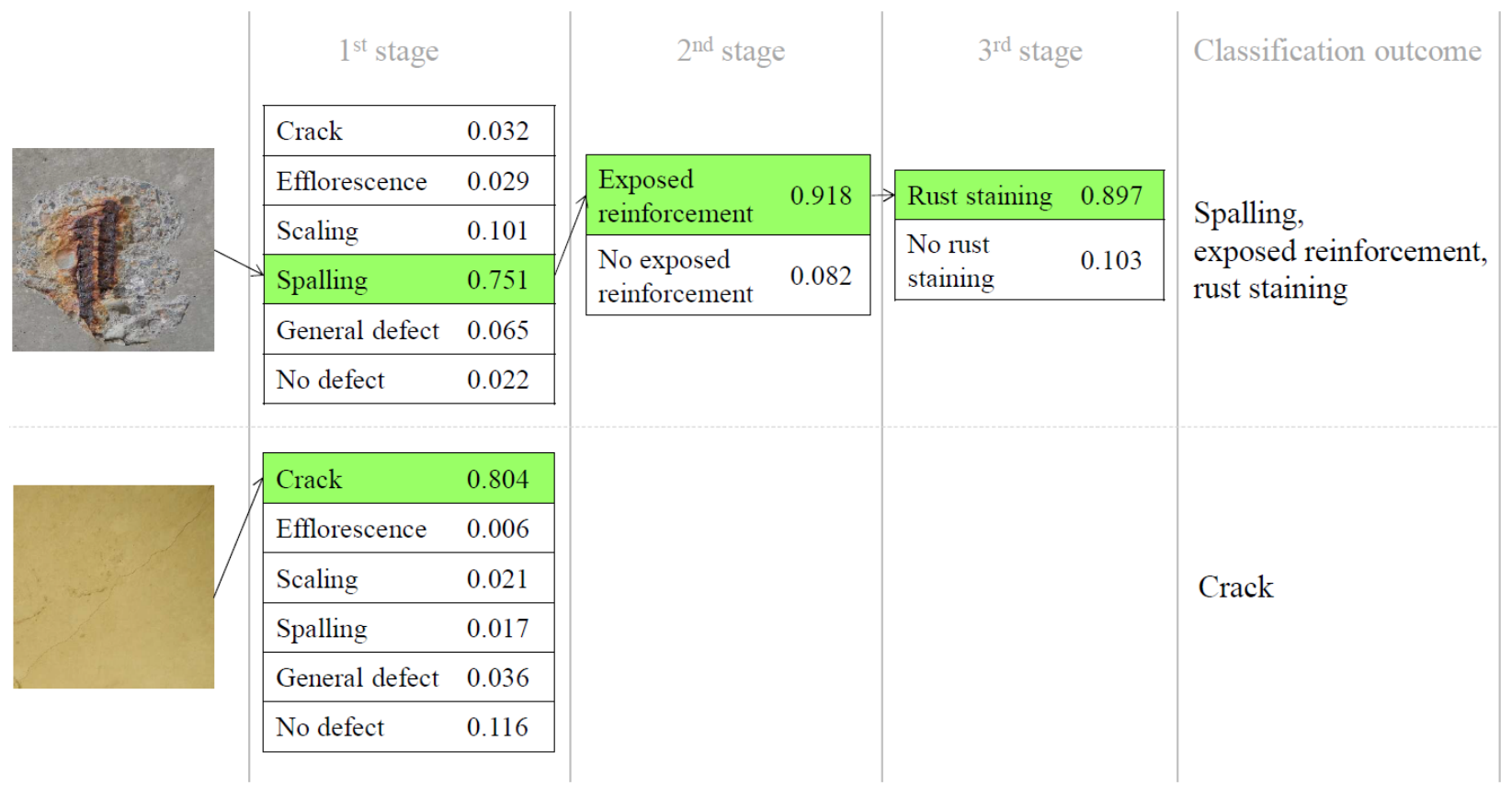

Figure 6 Demonstration of classification decision making

\section{Experiments and Results}

\subsection{Data requirements and equipment}

Data requirements arise from bridge inspection guidelines as well as from practical processing requirements. Existing inspection guidelines are developed for manual use as it happens today. There is no intent to use them for the automated data collection or processing. Hence, requirements must be converted from what is in the inspection guidelines, to something that is applicable for an automated process. Images collected in this study should meet the following major requirements:

Shooting range The data must be collected from different distances in order to take into account the sheer 
size of a bridge as well as the limited movement area. The minimum distance is typically the traffic-facing

248 side of a column where is very little space between the crash barrier and the support. Distance to the deck

249 ceiling or to columns in between traffic lanes is usually the maximal distance.

250 Resolution Two aspects must be considered when determining the required resolution: defects and features.

251 For defects, the lower size limit is a crack of $0.3 \mathrm{~mm}$. A resolution of 3 pixels per minimal feature minimises

252 the risk of under sampling. Hence, the on-surface resolution should be $0.1 \mathrm{~mm}$. For features, the resolution

253 must be enough to depict unique concrete surface details such as aggregate which can be identified again

254 in images taken from the same location but from a different position or a different point in time. The

255 resolution required by the error detection should suffice for this.

256 Focus Stably detecting features requires a sharp optical image with full utilization of the available 257 resolution. In addition, images are projected onto the surface as texture. As a result, blurred images lead to 258 a blurred texture. This complicates the later error detection.

259 Illumination Controlling illumination is not readily possible considering surrounding conditions (ongoing 260 traffic, weather, varying recording distance). Hence, limited lighting requirements are enjoined on the data 261 collection. The aim is to have a seamless surface texture with consistent lighting conditions. Limited 262 variations can be levelled and adjusted during post-processing. This means images should not depict strong 263 shadows. In addition, low lighting can occur below bridges, which requires a camera to work in such environments.

Surface angle The surface to optical camera axis angle should be as perpendicular to the surface as possible for two reasons: First, the depth of field is limited. Having a steep angle results in larger distance variance

267 from the camera to the surface and hence in parts of the surface image being out of focus. Secondly, no 268 optical lens is free from distortions. Structure-from-motion algorithms address this problem by estimating 269 and correcting the radial distortion. However, correcting these distortions is limited at steep surface angles.

270 The data requirements have direct implications on the camera selection. The resolution is an important 271 factor as it directly influences the surface resolution. In principle, even a camera with low resolution can 272 produce the same surface resolution, but in this case less surface area is covered per image and hence more 273 images are required. This complicates the collection of data. So, a higher resolution is beneficial. The 274 camera does not directly affect the lighting, but its sensitivity determines to what degree of illumination a 275 non-underexposed and non-overexposed image can be taken. Finally, the camera is a significant part of the 276 total cost, the price must be considered (budget for the equipment is £10,000). We chose Sony $\alpha 7 \mathrm{R}$ II for 277 the data collection in this research. The configurations of Sony $\alpha 7 \mathrm{R}$ II are: resolution 42 Megapixel, sensor 
size $36.0 \times 24.0 \mathrm{~mm}$, sensitivity $100-25,600$ ISO. Then, Illumination, shooting range and budget are the requirements to take into account for the lens selection. A coherent and complementary set of lenses were used: Sony 70-400mm F4.0-5.6 G2 ED SSM II, Sonnar T* FE 35mm F2.8 ZA Full-frame E-mount Lens, Canon EF 24-70mm f/2.8L II USM, and Canon EF 50mm f/1.4 USM.

\subsection{Dataset preparation}

There is no available open dataset which is sufficient in size and label quality that can be used for training a multi-classifier network. We used a total of 38,408 images out of which are 21,284 images from our own data collection of ten highway bridges around Cambridge plus 17,124 inspection images taken from bridge management systems which were kindly provided by authorities. We developed a software tool to simplify and speed up the data preparation process, which includes the manual extraction and manual labelling. We illustrate this tool in Figure 7. First, an image out of the raw image data set is presented to an operator. The image is inspected, and the defect area is identified and manually selected using a bounding box. The resulting image patch is extracted and visualized in a new window, from which the defect type can be selected by pressing a corresponding number on the keyboard. The image patch is then saved either in the training or validation folder. In addition to the defect classes, images were also randomly selected from the results of the previous step, i.e. Unhealthy Detection [5], to form a supplementary "No defect" class, as this is necessary to complete the training set and to identify images which were falsely suspected as being unhealthy. We demonstrate the process for extracting the defect samples for the multi-classifier in Figure 8.

The exposed reinforcement detector and the corrosion detector are both binary classifiers, meaning that they only have two classes. Thus, only two image sets were generated for each classifier: One showing the relevant condition and a complementary and balanced counterpart not showing the relevant condition. Having samples which are close to a boundary between two classes helps to increase the robustness of a classifier and especially helps to make a classifier sensitive to decisive features [33]. All defect images showing exposed reinforcement were used as the exposed reinforcement class. Spalling images and other defect images not showing exposed reinforcement were used as the counterpart. The dataset for the corrosion detector was constructed similarly, with all images showing corrosion building the corrosion dataset and other random defect images forming the no corrosion dataset. 







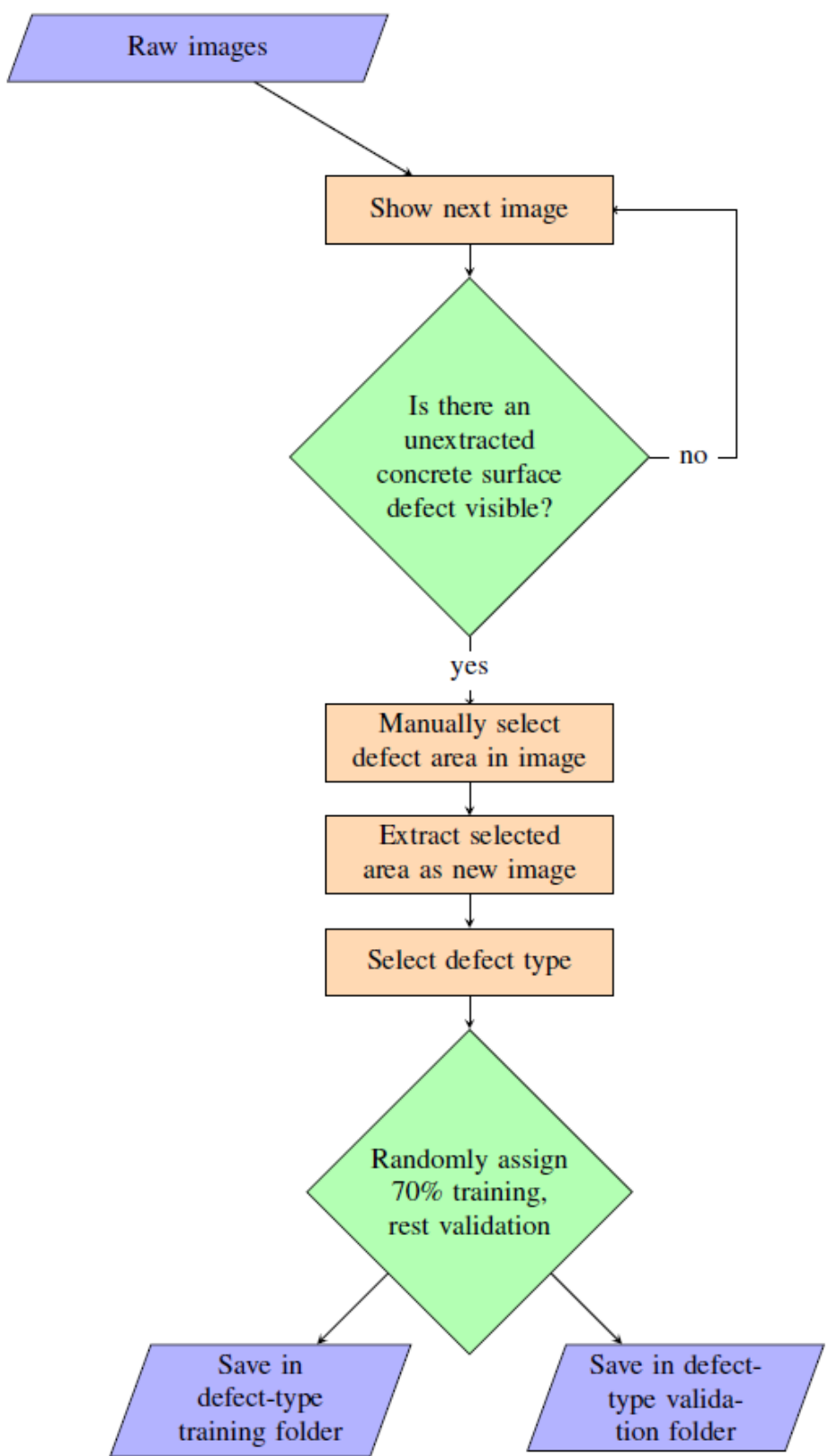

Figure 8 Extraction and labelling of training and validation dataset for multi-classifier

317 Each class in a training set needs to be approximately equal in size. Very unbalanced training datasets lead

318 to a classification bias towards the oversized class [34]. Images are randomly assigned to either the training

319 or validation set, typically using a $70 \%$ to $30 \%$ ratio. The training portion is larger than the validation set

320 based on the assumption that more training samples generally lead to a better model, whereas the validation

321 dataset still must be sufficient in size and variety to be meaningful in terms of representing defect variety.

322 Overall, 3,607 single defectimages were extracted based on 38,408 raw images. The number of extracted

323 defect images was drastically lower than the raw images because many of the images collected only show

324 intact concrete surfaces without a defect. Furthermore, many images out of the collection of authorities 
show defects not affecting the concrete structure itself but also affecting embankments, road surface or railing. Table 3 breaks down the number of images per defect class.

Table 3 Multi-Classifier training and validation dataset breakdown

\begin{tabular}{llccc}
\hline Classifier stage & Class label & $\begin{array}{c}\text { Training } \\
\text { samples }\end{array}$ & $\begin{array}{c}\text { Validation } \\
\text { samples }\end{array}$ & $\begin{array}{c}\text { Sum class } \\
\text { samples }\end{array}$ \\
\hline \multirow{3}{*}{ Multi-classifier } & Cracks & 551 & 238 & 789 \\
& Efflorescence & 202 & 109 & 311 \\
& General defect & 195 & 69 & 264 \\
& No defect & 339 & 113 & 452 \\
& Scaling & 116 & 52 & 168 \\
& Spalling & 306 & 121 & 427 \\
Exposed reinforcement & Exposed reinforcement & 156 & 67 & 223 \\
& No exposed reinforcement & 147 & 56 & 203 \\
\hline \multirow{2}{*}{ Corrosion Detector } & Rust staining & 243 & 112 & 355 \\
& No rust staining & 290 & 125 & 415 \\
\hline Total & & 2545 & 1062 & 3607 \\
\hline
\end{tabular}

\subsection{Implementation and network training}

329 The number of defect samples is not sufficient to train a deep neural network from scratch. To this end, we 330 applied a cross-learning strategy. A general dataset pre-trained network was used and fine-tuned on the 331 specific defect dataset. There are two strategies to fine-tune the network weights: the first is to only update 332 the weights of the final layer of the network, and the second is to propagate the error back through the whole 333 network. Just updating the final layer of the network requires less training time and memory while updating 334 the whole network leads to a higher classification reliability. An Inception V3 network was used as the deep 335 neural network for the state-of-the-art image classification [35]. The network consists of multiple stacked 336 inception modules. Each inception module consists of three or four parallel convolution operations with 337 varying filter sizes and one max or average pooling step. The convolution operation with a filter responds 338 to specific features of interest which are trained throughout the training process. Max and average pooling are steps to down-sample the network resolution and thus to train the number of variables. The network roughly needs five billion multiply-adds per inference and has less than 25 million parameters.

341 We used Gygax as the research platform to analyse and visualize the results [36]. Three separate network 342 instances were trained. The first stage (multi-classifier) had a total of seven output nodes: six for each of 343 the classes targeted by this study, and one as a background class. Using background class is common in 344 machine learning to represent inputs that do not fit into any of the desired classes. Note that this seventh 
class was not specifically trained, and it had negligibly small classification scores. Each instance was finetuned using an ImageNet pre-trained model and a batch-size of 32, an initial learning rate of 0.001 and a learning rate decay factor of 0.16 as hyper-parameters. All three networks converged within 300,000

348 training steps (Figure 9). Cross entropy is a function in information theory to quantify the distance between 349 a true, underlying distribution $p$ and a classification result $q$ [37]. Cross entropy $H$ was then calculated 350 using Formula (6. Minimizing the cross-entropy distance of a classifier maximises its predictive correctness.

$$
H(p, q)=-\sum_{x} p(x) \log q(x)
$$

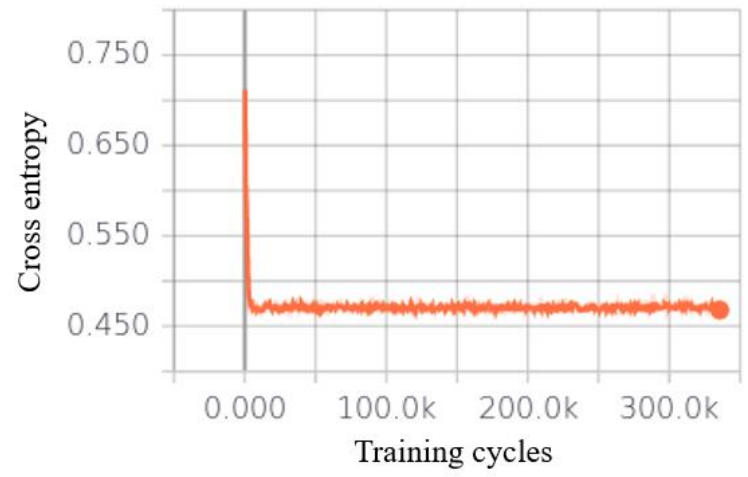

(a) Multi-classifier

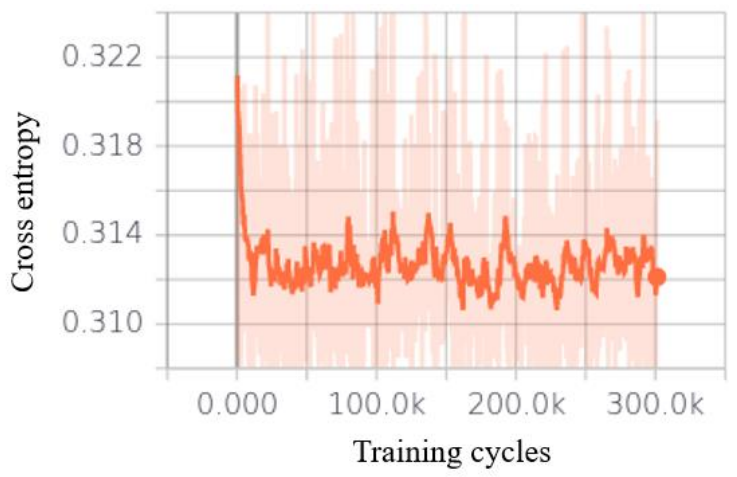

(b) Exposed reinforcement

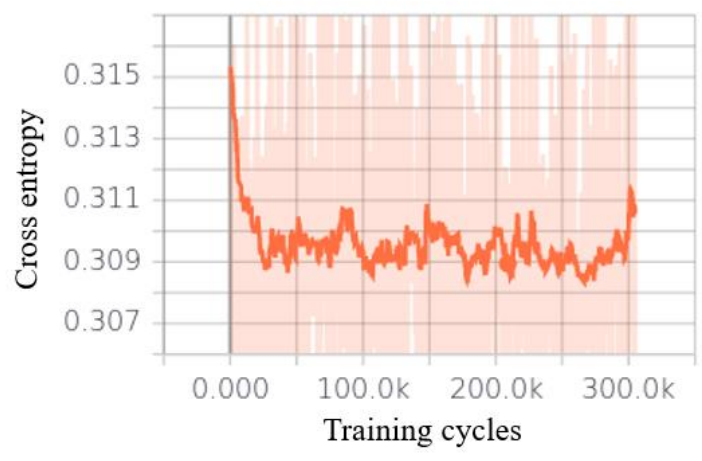

\section{$354 \quad 4.4$ Results}

355 The validation test set was classified and analysed quantitatively and qualitatively to demonstrate the 356 performance of the presented approach. It should be noted that validation images are not part of the training 
set to make sure the presented results are not influenced by over-fitting. Training and validation happened only once; images were not resorted.

\subsubsection{Multi-classifier}

Table 4 presents the confusion matrix for the first stage multi-classifier where the rows represent the manually assigned label and the columns represent the actual classification outcome. The quotient is calculated by the number of correctly classified images divided by the overall images in that particular class (sensitivity). The following is a discussion of the results, with a particular emphasis on discussing cases that were correctly classified. Subsets of the correctly classified examples are listed in Appendix for the sake of completeness.

Table 4 Multi-Classifier confusion matrix

\begin{tabular}{|c|c|c|c|c|c|c|c|}
\hline & \multicolumn{6}{|c|}{ Actual labels } \\
\hline & & Cracks & Efflorescence & Scaling & Spalling & General & No defect \\
\hline \multirow{6}{*}{  } & Cracks & $97.5 \%$ & $2.5 \%$ & $0.0 \%$ & $0.0 \%$ & $0.0 \%$ & $0.0 \%$ \\
\hline & Efflorescence & $1.8 \%$ & $90.8 \%$ & $0.9 \%$ & $1.8 \%$ & $0.0 \%$ & $4.6 \%$ \\
\hline & Scaling & $0.0 \%$ & $0.0 \%$ & $48.1 \%$ & $30.8 \%$ & $17.3 \%$ & $3.8 \%$ \\
\hline & Spalling & $4.1 \%$ & $1.7 \%$ & $5.0 \%$ & $86.0 \%$ & $1.7 \%$ & $1.7 \%$ \\
\hline & General & $5.8 \%$ & $4.3 \%$ & $5.8 \%$ & $4.3 \%$ & $72.5 \%$ & $7.2 \%$ \\
\hline & No defect & $0.9 \%$ & $0.9 \%$ & $0.0 \%$ & $1.8 \%$ & $1.8 \%$ & $94.7 \%$ \\
\hline
\end{tabular}

Defect classes cracks, efflorescence, and no defect were reliably detected with more than $90 \%$ sensitivity. The only misclassification in the class cracks happened to be efflorescence which is becoming clear when looking at the six misclassified images in Figure 10a. All images showed clear indication of efflorescence with white material building up next to the crack. This example illustrates the difficulty for inspectors to make an unambiguous decision in a case where both defects exist. The guidelines usually require the inspector to only document a single, predominant defect. It is thus an individual decision that which type of defect is documented according to the existing inspection scheme.

374 For the efflorescence defect class, 10 (or 9.2\%) of the defect samples were misclassified: 5 as no defect, 2 as cracks, one as scaling, and one as spalling. They are depicted in Figure 10b. It is noticeable that the first four misclassified images explicitly show stalactites that have formed over time from dissolved salt in dripping water. One possible explanation for the misclassification is an inadequate representation of such structures in the training data. The image in the middle was misclassified as a crack. In this case, as in the previous description for incorrectly classified cracks, both efflorescence and cracking were present. The 
with low contrast and poor image quality. There is a smooth transition from concrete showing normal discolouration towards the first signs of efflorescence.

Classifying spalling and scaling correctly is clearly a challenging task for the proposed classifier. There is a tendency towards spalling which can be explained by the unbalance in the training data towards spalling as can be seen from Table 3. Nevertheless, distinguishing between these two defect types is particularly error prone, and mutual misclassifications occurred frequently between these two classes. If viewing them as one combined class, however, the detection rate increased to $87.3 \%$. This can be explained by the fact that spalling and scaling appear almost identical, especially in the case of minor severity where it is difficult to distinguish even for a human eye. It sometimes depends on whether a single aggregate is shattered such that it can check if a defect was induced from an expanding aggregate (spalling) or from poor mortar losing its bond (scaling). In addition, the scaling class was underrepresented in the training data as no more examples were available. A subset of spalls classified as scaling is depicted in Figure 10c, scaling classified as spalling is depicted in Figure 10d.

Classifying defects in the general class is a challenging task due to the breadth of possible appearances: i.e. defects within this class are defined broadly as those that are not within the no defect class but are also not members of any of the specific defect classes. Hence, generalisation is difficult to achieve as this class covers the biggest range of possible textures. In addition, the number of training samples should not outnumber the other classes in order to preserve training balance. Still, the classifier correctly classified $72.5 \%$ of the validation examples. It should be noted that some of these general defect types were not included in the training data at all. The examples which were falsely classified as another defect are depicted in Figure 11a, the ones misclassified as no defect in Figure 11b.

As a last class, $94.7 \%$ out of the no defect validation samples were classified correctly. The ones that were misclassified are depicted in Figure 12. No general pattern for these misclassifications was noticeable.

Classification quality measures in a one-versus-all manner are listed in Table 5 in addition to the abovementioned class-by-class discussion. A given class was evaluated against all remaining classes. Again, the decline in classification quality was striking, especially for scaling, but also for the classes spalling and general. Adjusting the training data is the main option to influence where the line is drawn between classes. This again underlines the urgency to be particularly careful in the compilation of training data. 
413

414

415

416
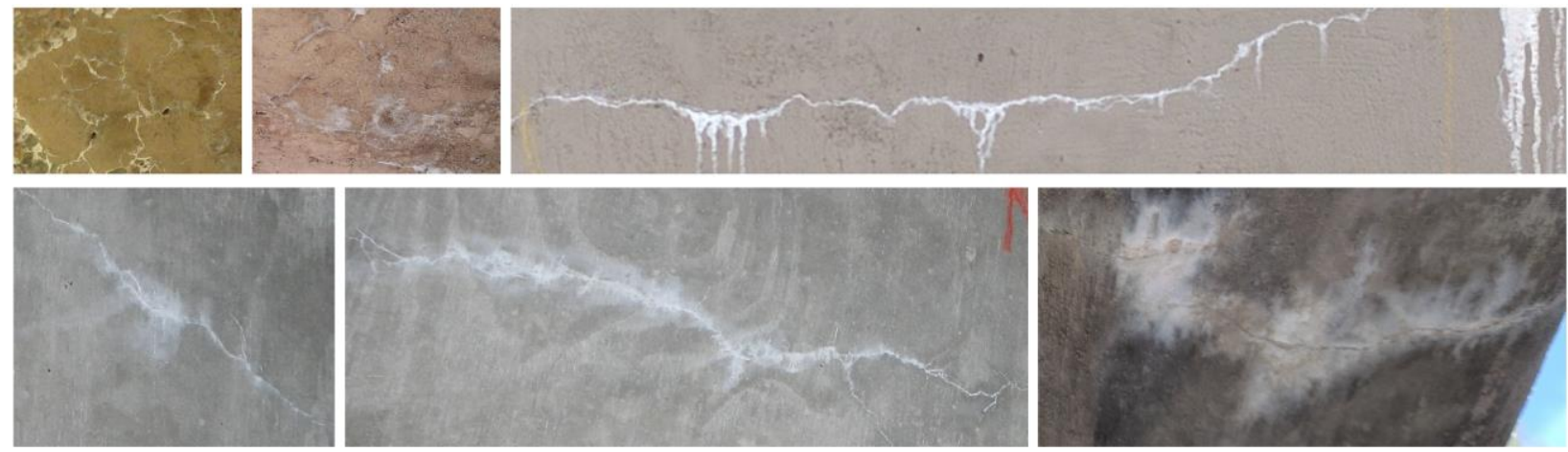

(a) Misclassified cracks as efflorescence
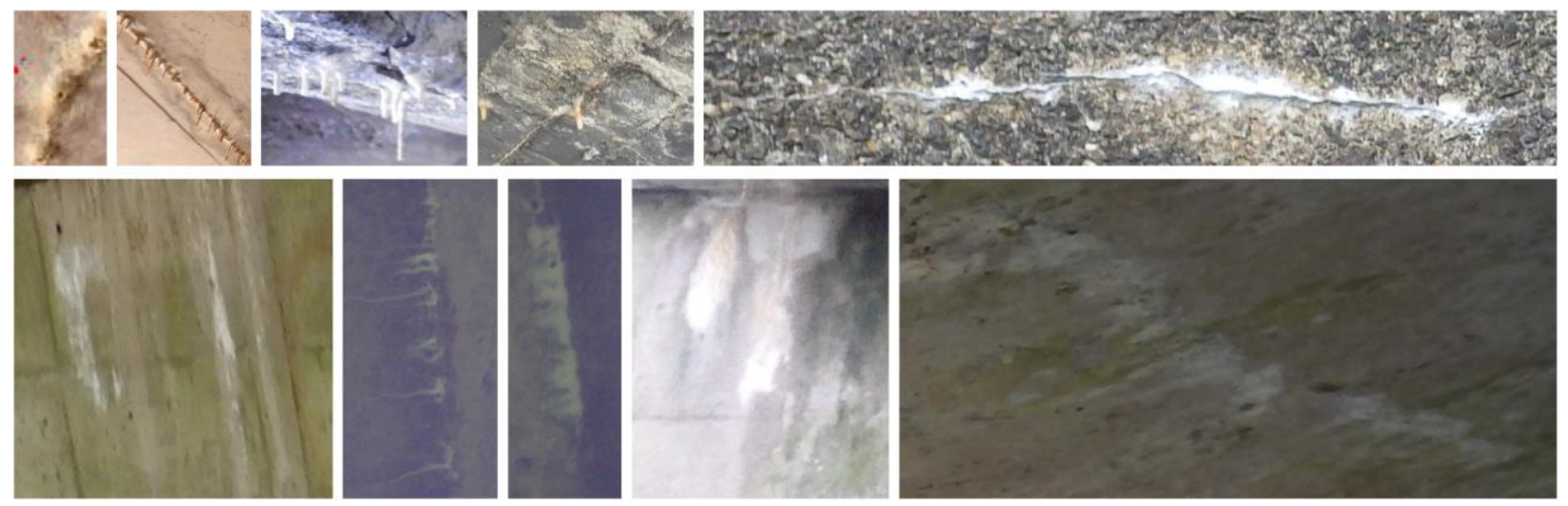

(b) Misclassified efflorescence as other defects (upper 5) and no defect (bottom 5)
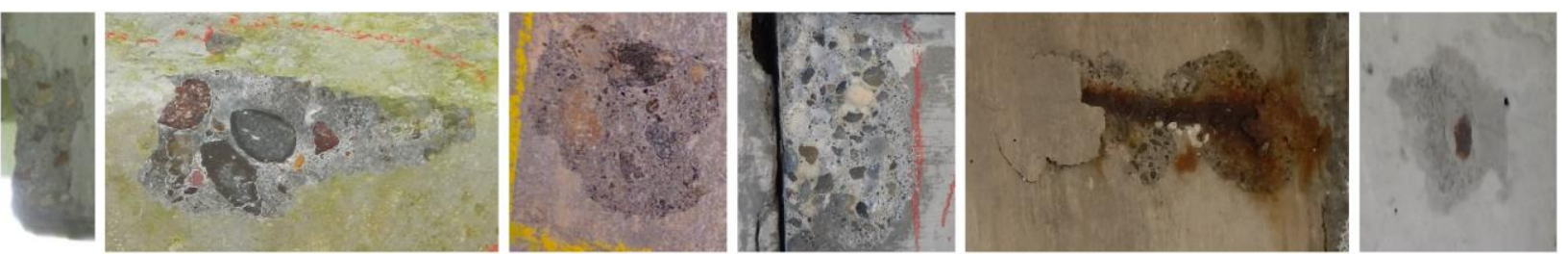

(c) Misclassified spalling as scaling (subset)


(d) Misclassified scaling as spalling (subset) 

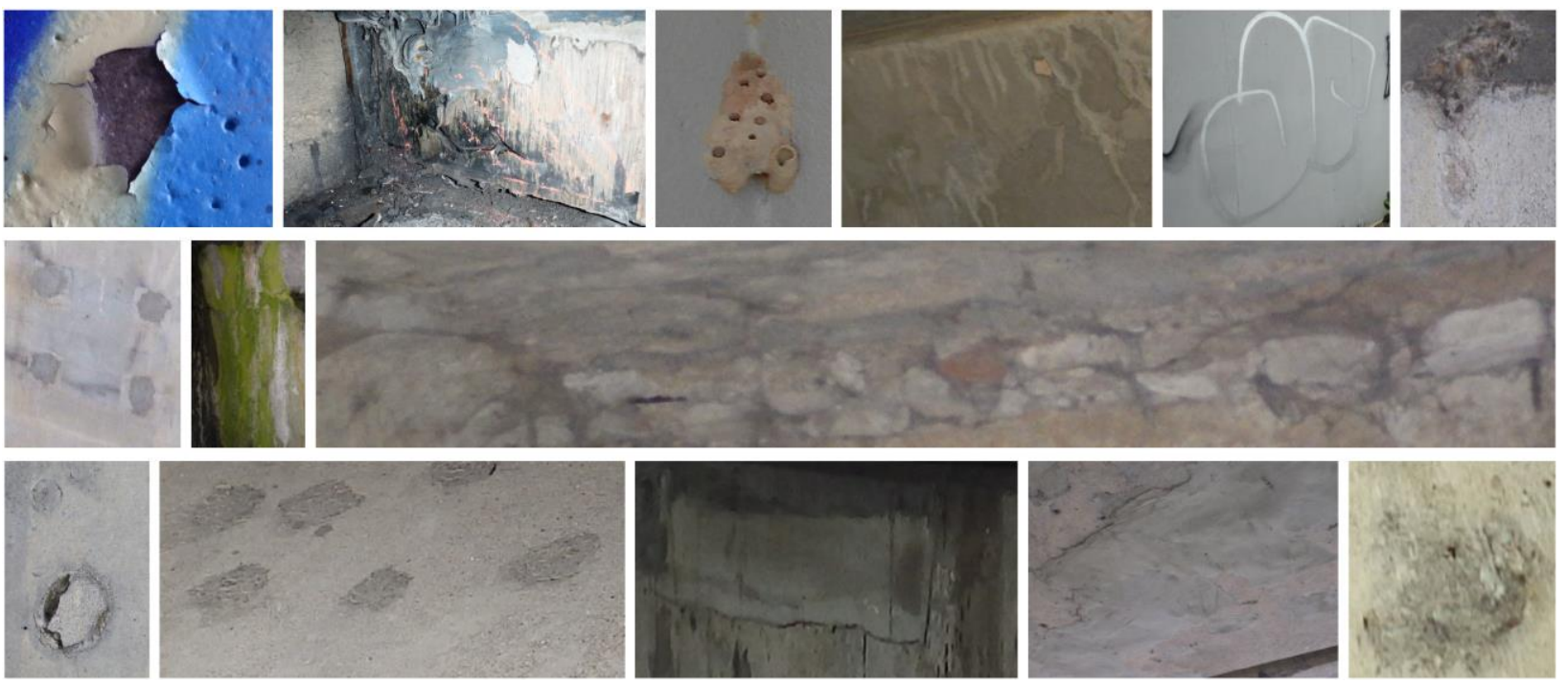

(a) Misclassified general as efflorescence
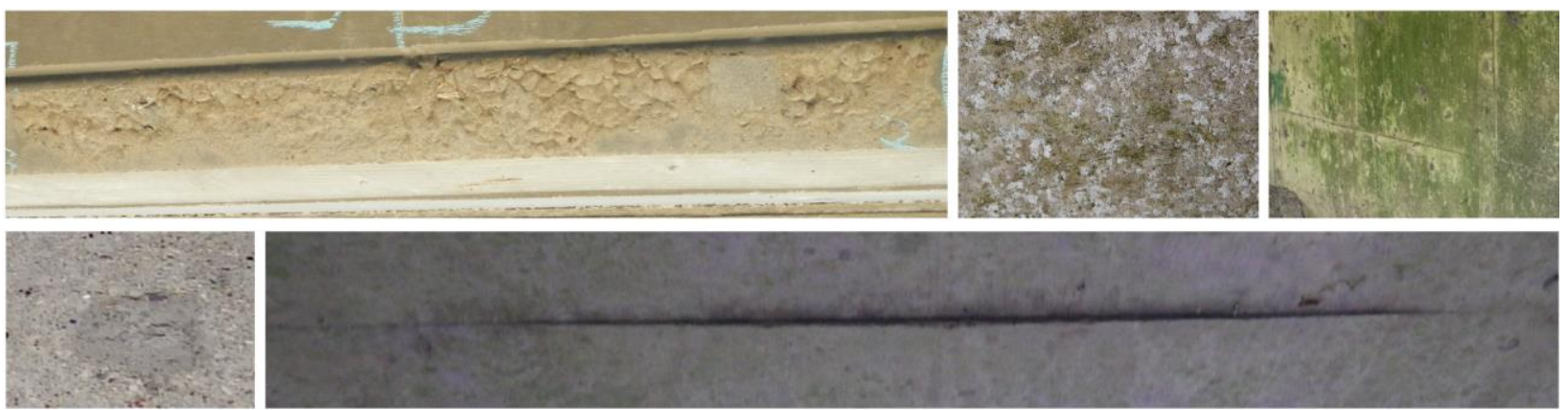

(b) Misclassified general as no defect


Figure 12 Examples of misclassified images of type no defects as a defect 
Table 5 Classification quality measures for multi-classifier

\begin{tabular}{lccccccc}
\hline Measure & Cracks & Efflorescence & Scaling & Spalling & General & No defect & $\begin{array}{c}\text { Spalling } \\
\text { Scaling }\end{array}$ \\
\hline TP & 232 & 99 & 25 & 104 & 50 & 107 & 151 \\
TN & 452 & 581 & 639 & 558 & 620 & 575 & 517 \\
FP & 12 & 12 & 11 & 23 & 13 & 14 & 12 \\
FN & 6 & 10 & 27 & 17 & 19 & 6 & 22 \\
Precision & $95.1 \%$ & $89.2 \%$ & $69.4 \%$ & $81.9 \%$ & $79.4 \%$ & $88.4 \%$ & $92.6 \%$ \\
Sensitivity & $97.5 \%$ & $90.8 \%$ & $48.1 \%$ & $86.0 \%$ & $72.5 \%$ & $94.7 \%$ & $87.3 \%$ \\
Specificity & $97.4 \%$ & $98.0 \%$ & $98.3 \%$ & $96.0 \%$ & $97.9 \%$ & $97.6 \%$ & $97.7 \%$ \\
Accuracy & $97.4 \%$ & $96.9 \%$ & $94.6 \%$ & $94.3 \%$ & $95.4 \%$ & $97.2 \%$ & $95.2 \%$ \\
$F_{1}$ & $96.3 \%$ & $90.0 \%$ & $56.8 \%$ & $83.9 \%$ & $75.8 \%$ & $91.5 \%$ & $89.9 \%$ \\
\hline
\end{tabular}

\subsubsection{Exposed reinforcement detector and corrosion detector}

439 We discuss in this section the classification quality for the exposed reinforcement detector and corrosion detector in a similar manner to the multi-classifier analysed in the preceding paragraphs. Table 7 presents the confusion matrix for exposed reinforcement, and Table 7 presents the quality measures. The detector reliably identified the presence of exposed reinforcement in an image with $91.2 \%$ sensitivity and correctly classified no exposed reinforcement as such with $81.8 \%$ sensitivity. Thus, the presence of exposed reinforcement was more reliable than the absence. Looking at the misclassified exposed reinforcement examples in Figure 13a, and no exposed reinforcement examples in Figure 13b respectively, it is clear that there are more variations of no exposed reinforcement which might appear as exposed reinforcement, e.g. shadows with a straight-line pattern, that look like exposed reinforcement. In contrast, the misclassified exposed reinforcement examples either show only a minor area in the image with reinforcement exposure, or the exposed reinforcement lies within a shadow. Both limitations can be addressed with better input imagery. Table 7 shows the quality measures for the exposed reinforcement detector with an $F_{l}$ score of $88.6 \%$.

452 Compared to the exposed reinforcement detection, the detection of rust staining was more reliable for both 453 the detection of presence with $94.6 \%$ sensitivity and the detection of absence with $95.2 \%$ sensitivity (Table 454 8). For the scope of this work, rust staining is both corrosion of exposed reinforcement as well as bleeding; 455 two differing patterns which must be learned from the classifier. The misclassified rust staining examples 456 (Figure 14) showing exposed reinforcement were affected by minor corrosion which might lead to the 457 misclassification. The misclassified examples not showing exposed reinforcement present corrosion 458 patterns in an atypical way (missing of typical drop / flowing down pattern). A regularity was not indicated 
from misclassified no rust staining examples. Table 9 lists the quality measures for the rust staining detector 460 with an $F_{l}$ score of $94.6 \%$.

Table 6 Exposed reinforcement detector confusion matrix

\begin{tabular}{|c|c|c|c|}
\hline & \multicolumn{2}{|c|}{ Actual labels } \\
\hline & & $\begin{array}{l}\text { Exposed } \\
\text { reinforcement }\end{array}$ & $\begin{array}{l}\text { No exposed } \\
\text { reinforcement }\end{array}$ \\
\hline \multirow{2}{*}{ 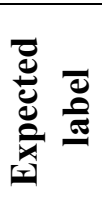 } & $\begin{array}{l}\text { Exposed } \\
\text { reinforcement }\end{array}$ & $91.2 \%$ & $8.8 \%$ \\
\hline & $\begin{array}{l}\text { No exposed } \\
\text { reinforcement }\end{array}$ & $18.2 \%$ & $81.8 \%$ \\
\hline
\end{tabular}

462

Table 7 Classification quality measures for exposed reinforcement detector

\begin{tabular}{lc}
\hline Measure & $\begin{array}{c}\text { Exposed } \\
\text { reinforcement }\end{array}$ \\
\hline TP & 62 \\
TN & 45 \\
FP & 10 \\
FN & 6 \\
Precision & $86.1 \%$ \\
Sensitivity & $91.2 \%$ \\
Specificity & $81.8 \%$ \\
Accuracy & $87.0 \%$ \\
$F_{1}$ & $88.6 \%$ \\
\hline
\end{tabular}

Table 8 Corrosion detector confusion matrix

\begin{tabular}{|c|c|c|c|}
\hline & \multicolumn{2}{|c|}{ Actual labels } \\
\hline & & $\begin{array}{c}\text { Rust } \\
\text { staining }\end{array}$ & $\begin{array}{l}\text { No rust } \\
\text { staining }\end{array}$ \\
\hline \multirow{2}{*}{ 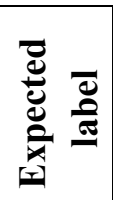 } & $\begin{array}{c}\text { Rust } \\
\text { staining }\end{array}$ & $94.6 \%$ & $5.4 \%$ \\
\hline & $\begin{array}{l}\text { No rust } \\
\text { staining }\end{array}$ & $4.8 \%$ & $95.2 \%$ \\
\hline
\end{tabular}




\begin{tabular}{lc}
\hline Measure & $\begin{array}{c}\text { Corrosion } \\
\text { detection }\end{array}$ \\
\hline TP & 106 \\
TN & 119 \\
FP & 6 \\
FN & 6 \\
Precision & $94.6 \%$ \\
Sensitivity & $94.6 \%$ \\
Specificity & $95.2 \%$ \\
Accuracy & $94.9 \%$ \\
$F_{1}$ & $94.6 \%$ \\
\hline
\end{tabular}

472

473

474
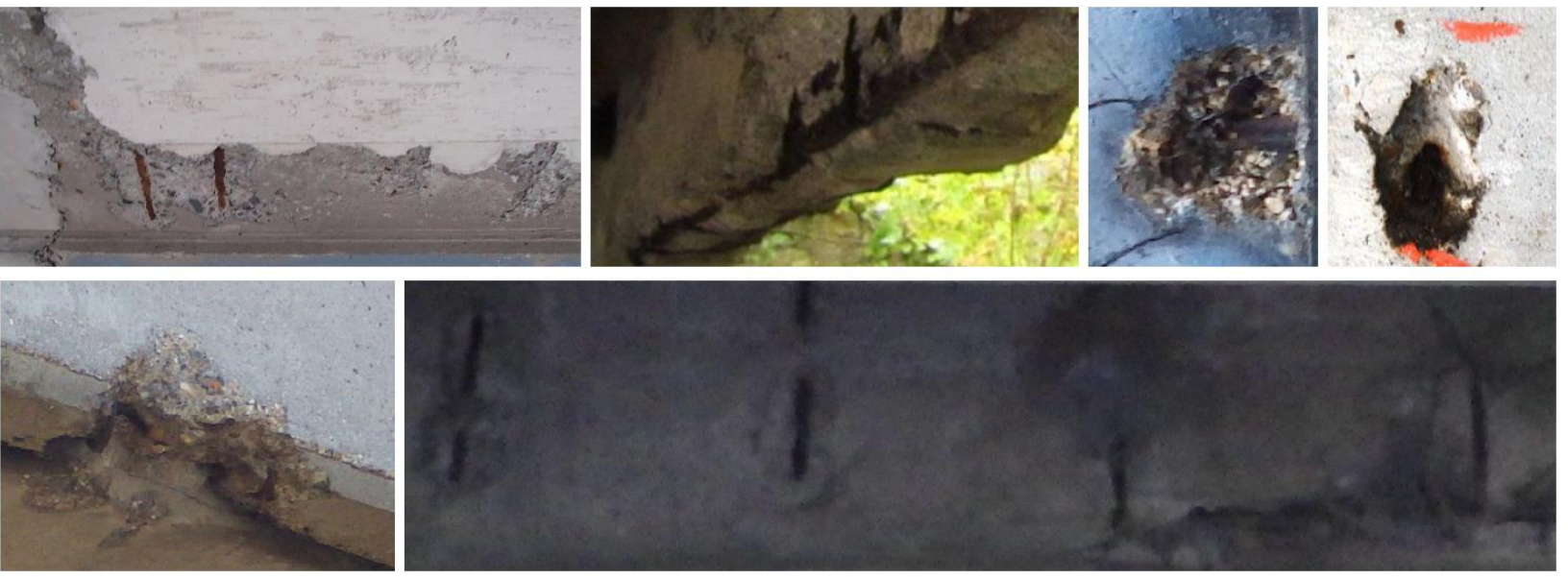

(a) Misclassified exposed reinforcement as no exposed reinforcement
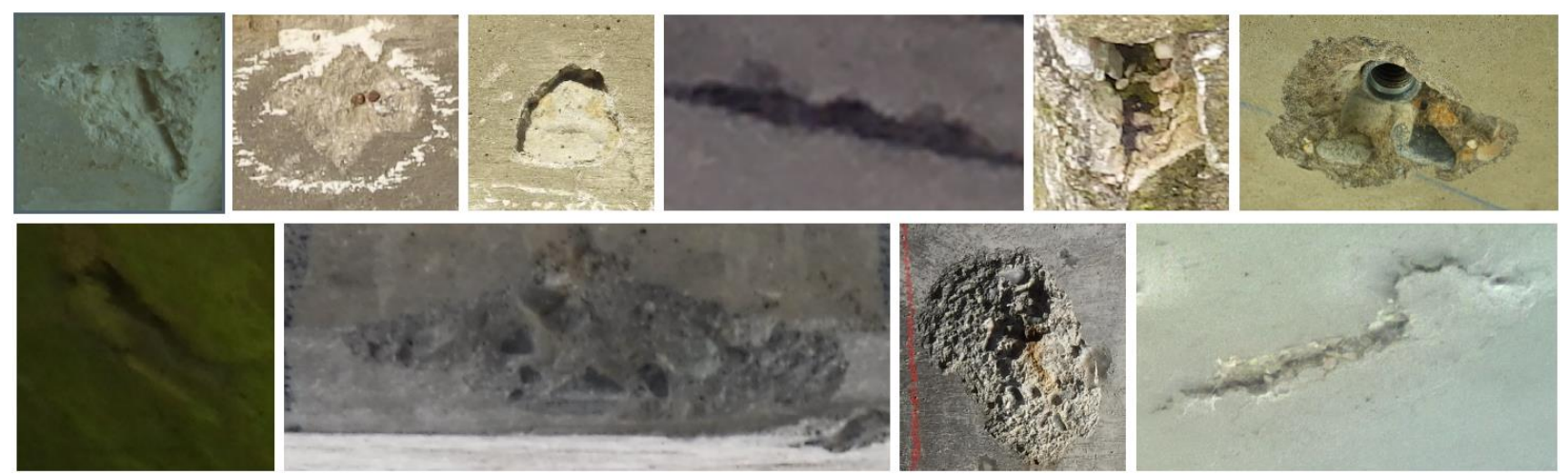

(b) Misclassified no exposed reinforcement as exposed reinforcement 

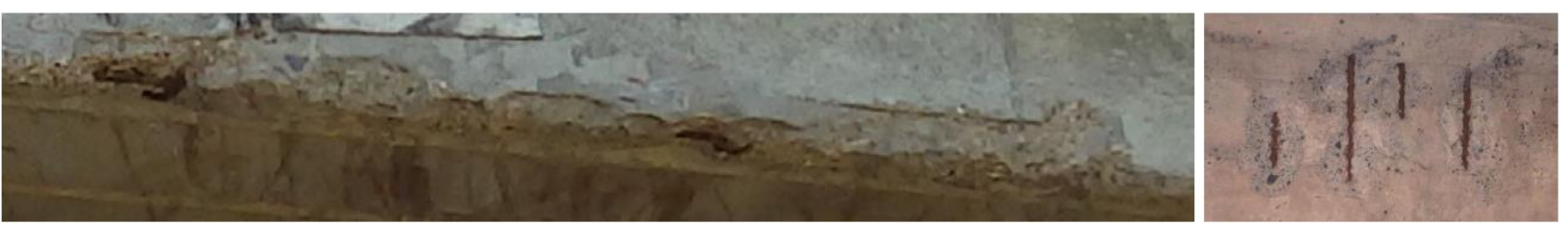

479
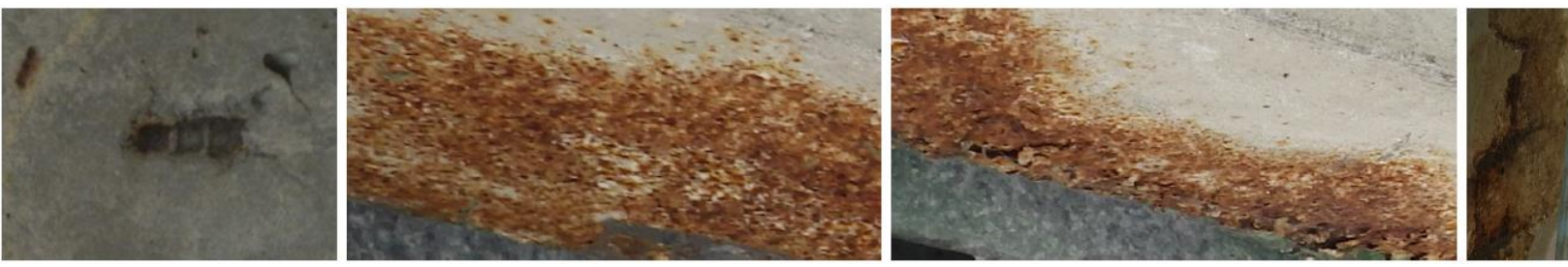

(a) Misclassified rust staining as no rust staining
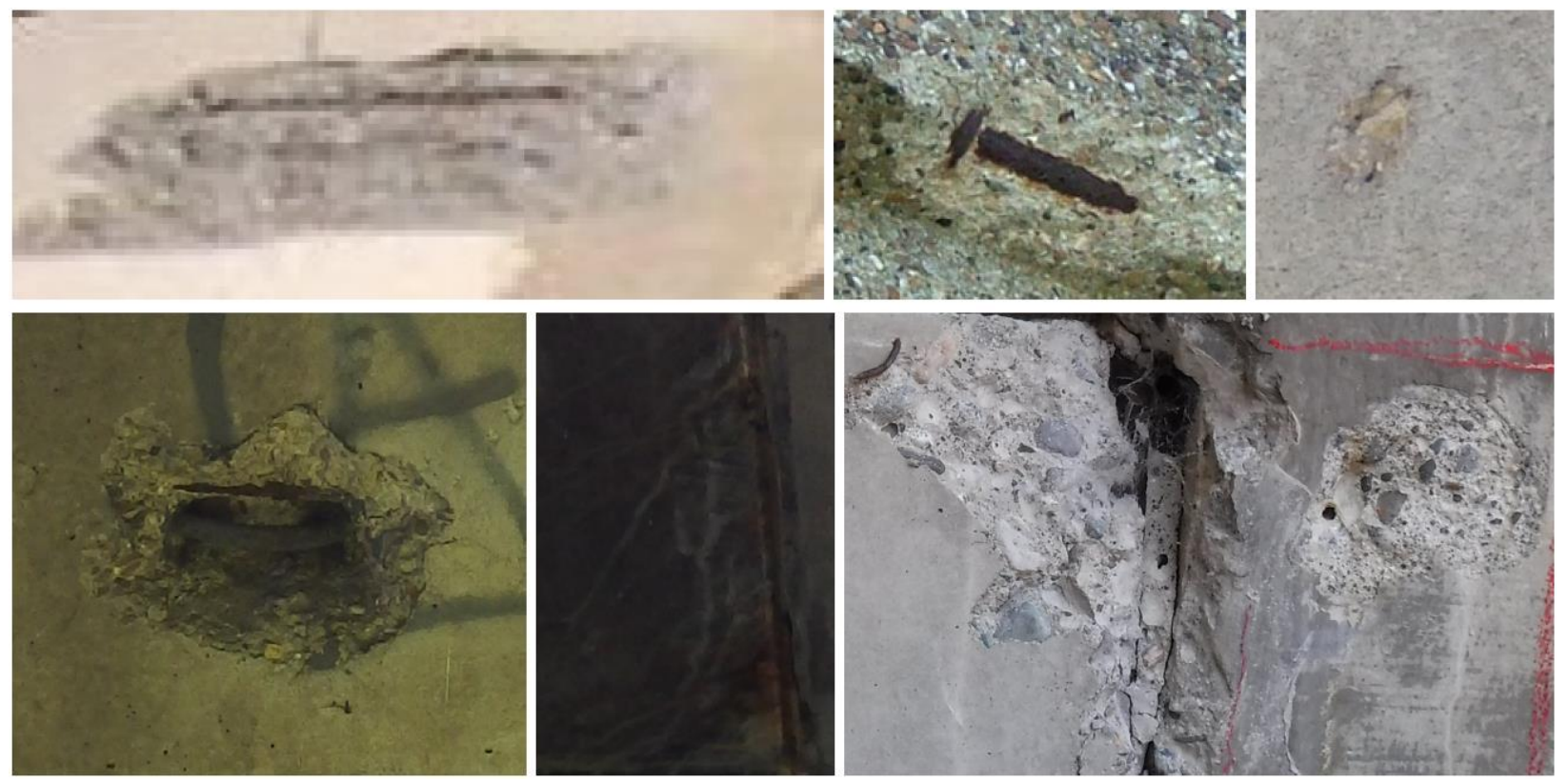

(b) Misclassified no rust staining as rust staining

Figure 14 Examples of misclassified rust staining

\section{Conclusionss}

We presented a multi-classifier that can assign none, one, or multiple classification labels to a defect image.

The possible defect variants were derived from inspection guidelines and could be adopted to such local variations. A hierarchical classification approach was presented that consists of three stacked image classifiers. These classifiers were trained using manually labelled training data from own data collection and from authority image sets. It was afterwards assessed using unseen validation data. Experimental results showed that the classifiers can reliably assign class labels to an image from a variety of classes with an 
average $F_{l}$ score of $83.5 \%$. This demonstrates that the three-staged hierarchical multi-classifier can reliably classify potentially unhealthy concrete surface patches into a defect classes in accordance with existing bridge inspection guidelines.

The limitations of the presented approach are clearly related to the training data. Some defect classes were underrepresented compared to others. This is the main reason why the multi-classifier caused misclassifications and bias. Only the availability of a large and correctly labelled dataset can lead to reliable classification results. In addition, as with machine learning in general, it is difficult to understand how a decision is derived. It is not transparent which exact features were used by the classifier to distinguish different defect. These limitations allow us to formulate solutions for future: using the dataset presented in this work as a starting point but adding more examples which are discussed and labelled by experts would lead to a reliable system on a broader base. In addition, visualising layer activations or deep dream could help to understand which elements of an image are used in the classification process. Using an automated classifier, despite its limitations, still has a key benefit: a decision is reproducible and can be repeated at any given time if improved training data or classifiers arise or if the inspection guidelines are changed with definition of different defect classes.

Practitioners must adjust to the significant changes that digitisation brings with it. This applies to those who are responsible for the development of the inspection guidelines as well as to those who apply the guidelines on site. Not all that is technically possible will be easily applied in everyday routine.

This work bridges the gap between the requirements arising from established inspection guidelines, the demand for holistic data model which has recently become known as "Digital Twin" (DT), and methods for automatically identifying and measuring specific defect classes on small scale images. This work, together with other work in this area, is however just the beginning of a longer effort to create toolsets needed to build the national DT roadmap.

From a wider perspective, it makes contributions to ensure the daily safety and availability of infrastructure to society. The availability of a comprehensive and open digital representation of the data required for bridge inspection is an indispensable necessity for exploiting the full potential of modern digital technologies like big data exploration, artificial intelligence, and database technologies. Only on this basis can experiences and information be exchanged and made available between various international maintenance authorities, and conclusions can be drawn automatically from previously found and applied solutions. 


\section{Acknowledgements}

524

525

526

527

528

529

530

531

532

533

534

535

536

537

538

539

This research work is supported by EPSRC, Trimble Inc., Cambridge Trimble Fund, and Infravation SeeBridge project under Grant Number No. 31109806.0007. SeeBridge is co-funded by Funding Partners of the ERA-NET Plus Infravation and the European Commission. The Funding Partners of the Infravation 2014 Call are: Ministerie van Infrastructuur en Milieu, Rijkswaterstaat, Bundesministerium fr Verkehr, Bauund Stadtentwicklung, Danish Road Directorate, Statens Vegvesen Vegdirektoratet, Trafikverket Trv, Vegagerin, Ministere de Lecologie, du Developpement Durable et de Lenergie, Centropara el Desarrollo Tecnologico Industrial, Anas S.P.A., Netivei Israel National Transport Infrastructure Company Ltd. and Federal Highway Administration USDOT. We would like to thank for their supports. Any opinions, findings, and conclusions or recommendations expressed in this material are those of the authors and do not necessarily reflect the views of EPSRC, Trimble, or Infravation SeeBridge. We also thank the representatives from Atkins UK, the U.S. Federal Highway Administration and Georgia DoT for supporting this research with parts of their bridge inspection image stock.

\section{Data Availability Statement}

The training and validation dataset of the multi-classifier can be accessed by the following link: https://doi.org/10.5281/zenodo.2601506.

\section{References}

[1] F. Xie, D. Levinson, Evaluating the effects of the I-35W bridge collapse on road-users in the twin cities metropolitan region, Transportation Planning and Technology. (2011). doi:10.1080/03081060.2011.602850.

[2] Department of Employment and Economic Development, Economic Impacts of the I-35W Bridge Collapse, Technical Report, (2007). http://www.dot.state.mn.us/\%0A398 i35wbridge/rebuild/pdfs/economic-impacts-from-deed.pdf, accessed 16 March 2019.

[3] Design Manual for Roads and Bridges, Inspection of Highway Structures 2007. Available at http://www.standardsforhighways.co.uk/ha/standards/dmrb/vol3/index.htm, accessed 16 March, 2019.

[4] R. Lu, I. Brilakis, C.R. Middleton, Detection of Structural Components in Point Clouds of Existing RC Bridges, Computer-Aided and Civil and Infrastructure Engineering (2018). doi:10.1111/mice.12407.

[5] P. Hüthwohl, I. Brilakis, Detecting healthy concrete surfaces, Advanced Engineering Informatics. (2018). doi:10.1016/j.aei.2018.05.004. 
[6] B.M. Phares, G.A. Washer, D.D. Rolander, B.A. Graybeal, M. Moore, Routine Highway Bridge Inspection Condition Documentation Accuracy and Reliability, Journal of Bridge Engineering. (2004). doi:10.1061/(ASCE)1084-0702(2004)9:4(403).

[7] U.S. Department of Transportation, NBI ASCII Files - National Bridge Inventory - Bridge Inspection - Safety - Bridges \& Structures - Federal Highway Administration, 2015. https://www.fhwa.dot.gov/bridge/nbi/ascii.cfm?year=2014, accessed 16 March, 2019

[8] B. u. S. Bundesministerium für Verkehr, Bauwerksprüfung nach DIN 1076, Technical Report, Bundesministerium für Verkehr, Bau und Stadtentwicklung, Berlin, (2013). https:/www.bmvi.de/SharedDocs/DE/Anlage/VerkehrUndMobilitaet/Strasse/dokumentationbauwerkspruefung-nach-din-1076.pdf, accessed 16 March, 2019

[9] U.S. Department of Transportation, Bridge Evaluation Quality Assurance in Europe, Technical Report, 2008. https://international.fhwa.dot.gov/pubs/p108016/p108016.pdf, accessed 16 March, 2019.

[10] ASCE, 2017 Infrastructure Report Card - Bridges, Technical Report, 2017. https://www.infrastructurereportcard.org/wp-content/uploads/2017/01/Bridges-Final.pdf, accessed 16 March, 2019.

[11] Department for Transport and Highways Agency, Maintaining Strategic Infrastructure: Roads, Technical Report, 2014. https://www.nao.org.uk/wp-content/uploads/2015/06/MaintainingStrategic-Infrastructure-Roads.pdf, accessed 16 March, 2019.

[12] M.B. Johnson, W. Casey, L. O'Donnell, P. Allec, A.R. Marshall, D. Soden, Caltrans Bridge Element Inspection Manual, Technical Report, 2016. http://www.dot.ca.gov/hq/structur/strmaint/elem_man.pdf, accessed 16 March, 2019.

[13] Queensland Department of Main Roads, Bridge Inspection Manual, Technical Report, 2004. https:/catalogue.nla.gov.au/Record/3424275, accessed 16 March, 2019.

[14] Alberta Infrastucture and Transportation, Bridge Inspection \& Maintenance System, Technical Report, 2008.

http://www.transportation.alberta.ca/Content/docType30/Production/BIMMnlv3.1.pdf, accessed 16 March, 2019.

[15] A. Kedar, S. Peretz, D. Stuchevsky, H. Fathi, R. Lu, P. Vela, D. Singer, R. Sacks, L. Ma, U. Kattel, I. Brilakis, P. Huethwohl, A. Borrmann, S. Daum, Automated Compilation of Semantically Rich BIM Models of Bridges - Information Delivery Manual, Technical Report, 2016. https://seebridge.net.technion.ac.il/reports/\#Reports, accessed 16 March, 2019.

[16] Leica Geosystems AG, Leica Viva TS11/TS15 KUMONOS The remote concrete crack measurement system, Technical Report, Heerbrugg, 2015. http://kumonos.info/downloads/brochure_kumonos_leica.pdf, accessed 16 March, 2019.

[17] Nikon Trimble, Technical Report, Building Construction Sales Department, Tokyo, 2017. https://www.nikon-trimble.co.jp/products/pdf/measurement/nivoi_crackdetection.pdf, accessed 16 March, 2019.

[18] P. Hüthwohl, I. Brilakis, A. Borrmann, R. Sacks, Integrating RC Bridge Defect Information into BIM Models, Journal of Computing in Civil Engineering. 32 (2018) 04018013. doi:10.1061/(ASCE)CP.1943-5487.0000744.

[19] E. E. Bisson, Friction, Wear, and the Influence of Surfaces, Technical Report, National Aeronautics and Space Administration, in: Washington D.C., 1968. https://ntrs.nasa.gov/archive/nasa/casi.ntrs.nasa.gov/19680004168.pdf, accessed 16 March, 2019.

[20] C.M. Yeum, J. Choi, S.J. Dyke, Autonomous image localization for visual inspection of civil infrastructure, Smart Materials and Structures. (2017). doi:10.1088/1361-665X/aa510e.

[21] C. Koch, K. Georgieva, V. Kasireddy, B. Akinci, P. Fieguth, A review on computer vision based defect detection and condition assessment of concrete and asphalt civil infrastructure, Advanced Engineering Informatics. (2015). doi:10.1016/j.aei.2015.01.008.

[22] Z.-D. Xu, X. Zeng, S. Li, Damage Detection Strategy Using Strain-Mode Residual Trends for Long-Span Bridges, Journal of Computing in Civil Engineering (2013). 
doi:10.1061/(asce)cp.1943-5487.0000371.

[23] G.K. Choudhary, S. Dey, Crack detection in concrete surfaces using image processing, fuzzy logic, and neural networks, in: 2012 IEEE 5th International Conference on Advanced Computational Intelligence. ICACI 2012, 2012. doi:10.1109/ICACI.2012.6463195.

[24] T. Yamaguchi, S. Nakamura, R. Saegusa, S. Hashimoto, Image-based crack detection for real concrete surfaces, IEEJ Transactions On Electrical and Electronic Engineering. (2008). doi:10.1002/tee.20244.

[25] I. Abdel-Qader, O. Abudayyeh, M.E. Kelly, Analysis of Edge-Detection Techniques for Crack Identification in Bridges, Journal of Computing in Civil Engineering (2003). doi:10.1061/(asce)0887-3801(2003)17:4(255).

[26] M.R. Jahanshahi, S.F. Masri, Adaptive vision-based crack detection using 3D scene reconstruction for condition assessment of structures, Automation in Construction, 2012. doi:10.1016/j.autcon.2011.11.018.

[27] A.M.A. Talab, Z. Huang, F. Xi, L. Haiming, Detection crack in image using Otsu method and multiple filtering in image processing techniques, Optik (Stuttg). (2016). doi:10.1016/j.ijleo.2015.09.147.

[28] P. Prasanna, K. Dana, N. Gucunski, B. Basily, Computer-vision based crack detection and analysis, in: Sensors and Smart Structures Technologies for Civil, Mechanical, and Aerospace Systems. 2012, 2012. doi:10.1117/12.915384.

[29] P. Prasanna, K.J. Dana, N. Gucunski, B.B. Basily, H.M. La, R.S. Lim, H. Parvardeh, Automated Crack Detection on Concrete Bridges, IEEE Transactions on Automation Science and Engineering. (2016). doi:10.1109/TASE.2014.2354314.

[30] M.R. Jahanshahi, F. Jazizadeh, S.F. Masri, B. Becerik-Gerber, Unsupervised Approach for Autonomous Pavement-Defect Detection and Quantification Using an Inexpensive Depth Sensor, Journal of Computing in Civil Engineering (2012). doi:10.1061/(asce)cp.1943-5487.0000245.

[31] S. German, I. Brilakis, R. DesRoches, Rapid entropy-based detection and properties measurement of concrete spalling with machine vision for post-earthquake safety assessments, Advanced Engineering Informatics. 26 (2012) 846-858. doi:10.1016/j.aei.2012.06.005.

[32] C. Feng, M.-Y. Liu, C.-C. Kao, T.-Y. Lee, Deep Active Learning for Civil Infrastructure Defect Detection and Classification, in: 2017. doi:10.1061/9780784480823.036.

[33] A.L. Blum, P. Langley, Artificial Intelligence Selection of relevant features and examples in machine learning, Artificial Intelligence (1997). doi:10.1016/S0004-3702(97)00063-5.

[34] M. Kubat, S. Matwin, Addressing the Curse of Imbalanced Training Sets: One-Sided Selection, in: Proceedings of the Fourteenth Internationl Conference on Machine Learning, 1997. doi:10.1007/s13398-014-0173-7.2.

[35] C. Szegedy, V. Vanhoucke, S. Ioffe, J. Shlens, Z. Wojna, Rethinking the Inception Architecture for Computer Vision, in: IEEE Conference on Computer Vision and Pattern Recognition, Las Vegas, NV, 2016: p. 2818-2826.

[36] P. Huethwohl, I. Armeni, H. Fathi, I. Brilakis, Gygax Construction IT research platform for 2D \& 3D, 2017. https://github.com/ph463/Gygax, accessed 16 March, 2019.

[37] D.M. Hutton, The Cross-Entropy Method: A Unified Approach to Combinatorial Optimisation, Monte-Carlo Simulation and Machine Learning, Kybernetes. (2005). doi:10.1108/03684920510595562. 


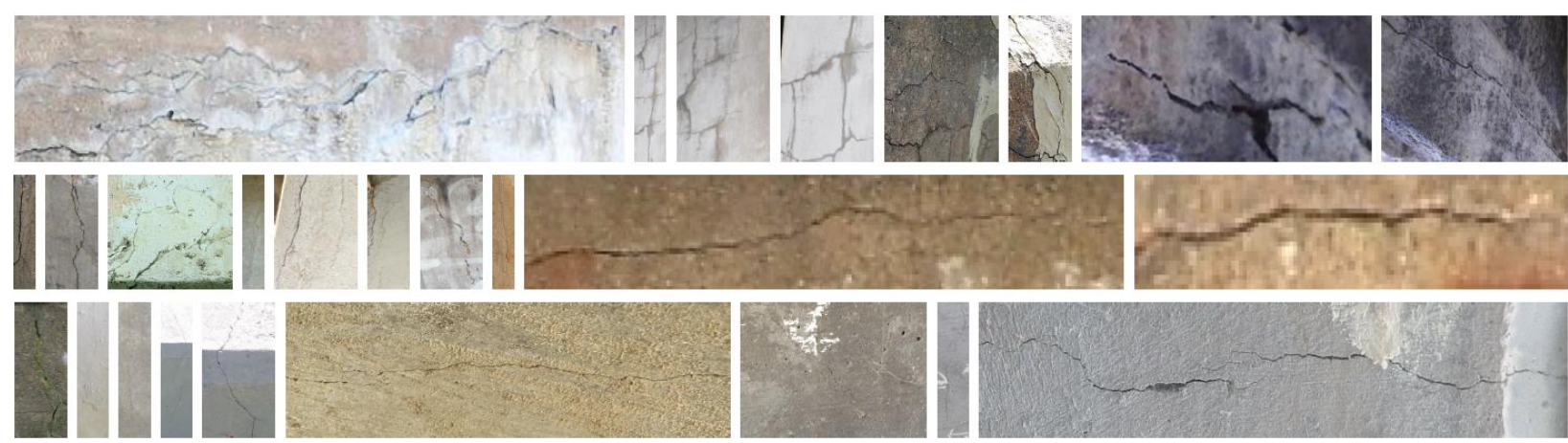

652

(a) Correctly classified images of class cracks (subset)

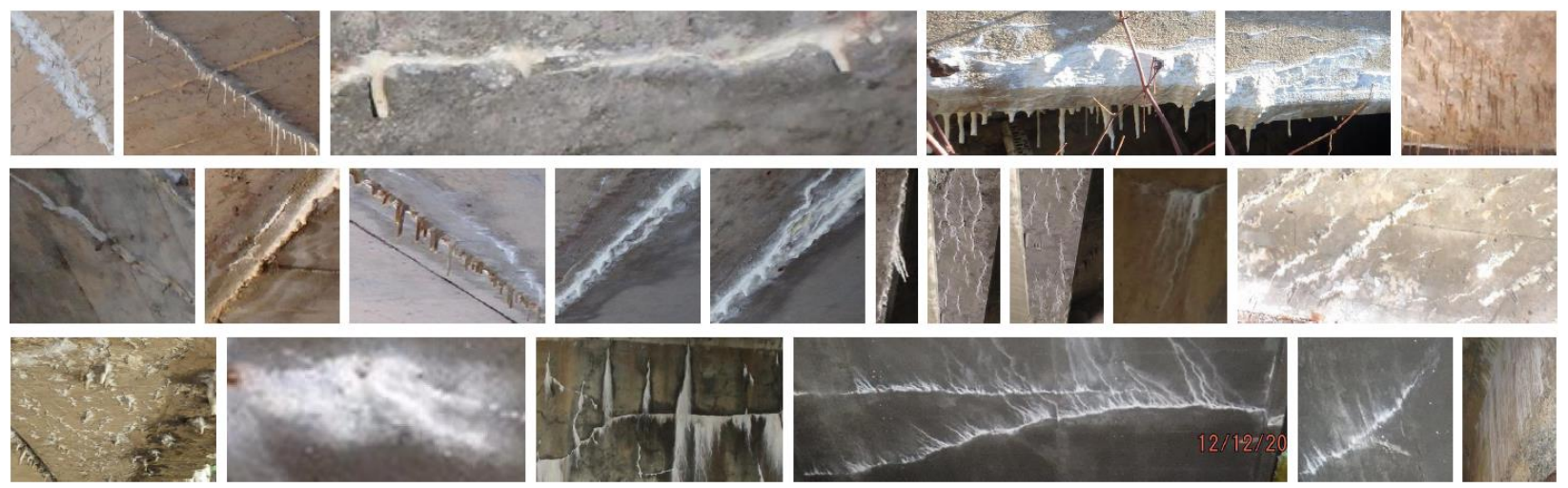

(b) Correctly classified images of class efflorescence (subset)
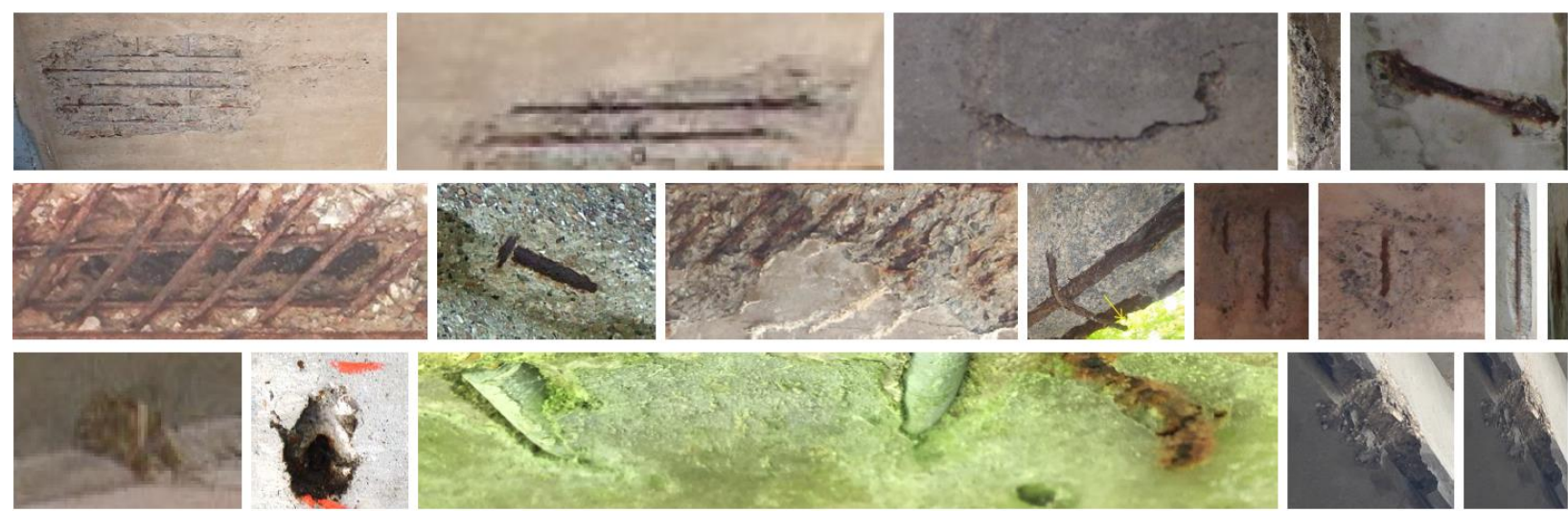

(c) Correctly classified images of class spalling (subset) 



(d) Correctly classified images of class scaling (subset)
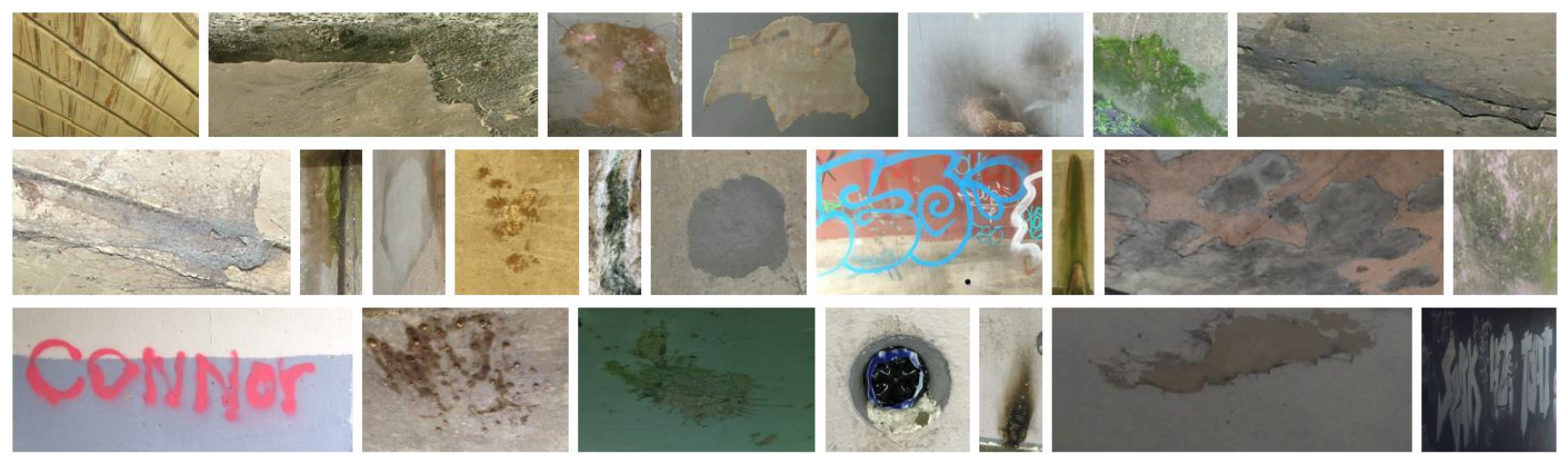

(e) Correctly classified images of class scaling (subset)
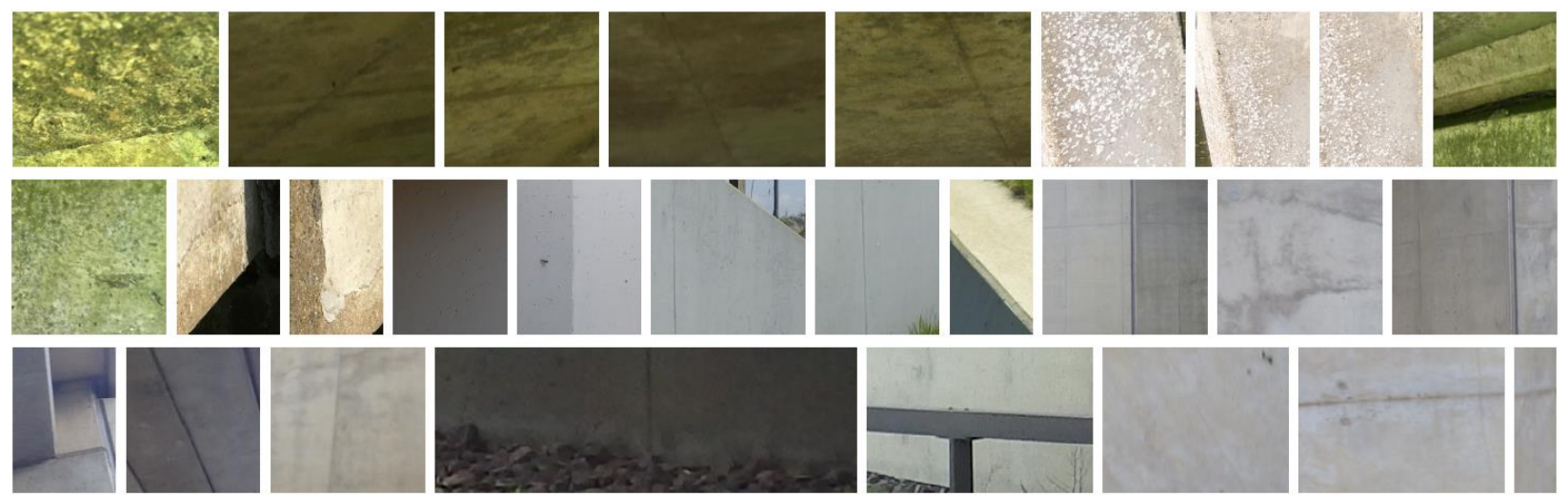

662

(f) Correctly classified images of class no defect (subset) 

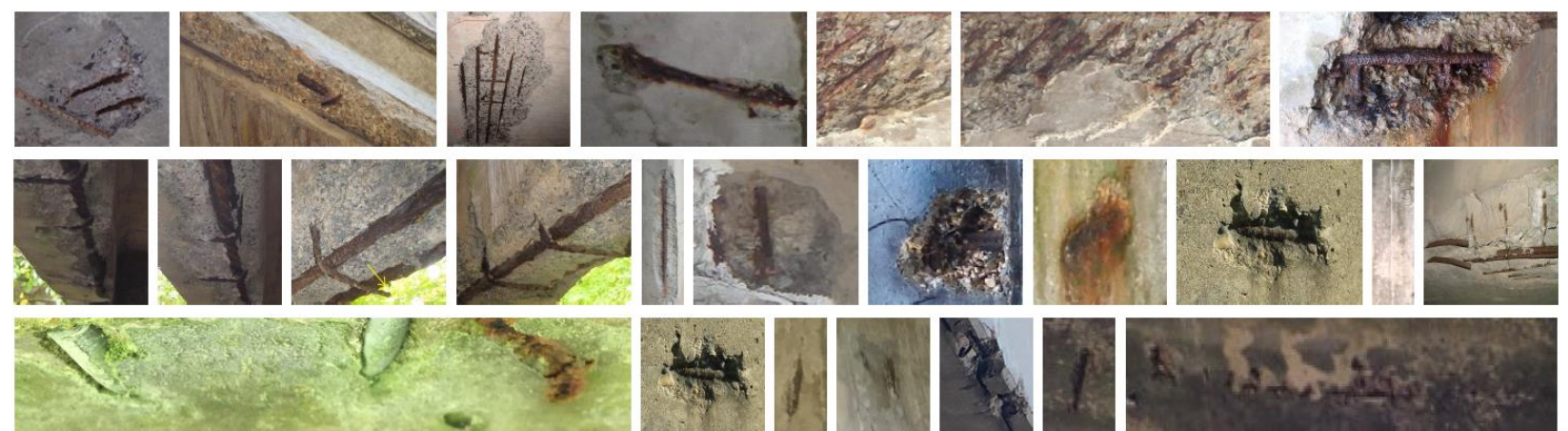

(g) Correctly classified images of class exposed reinforcement (subset)

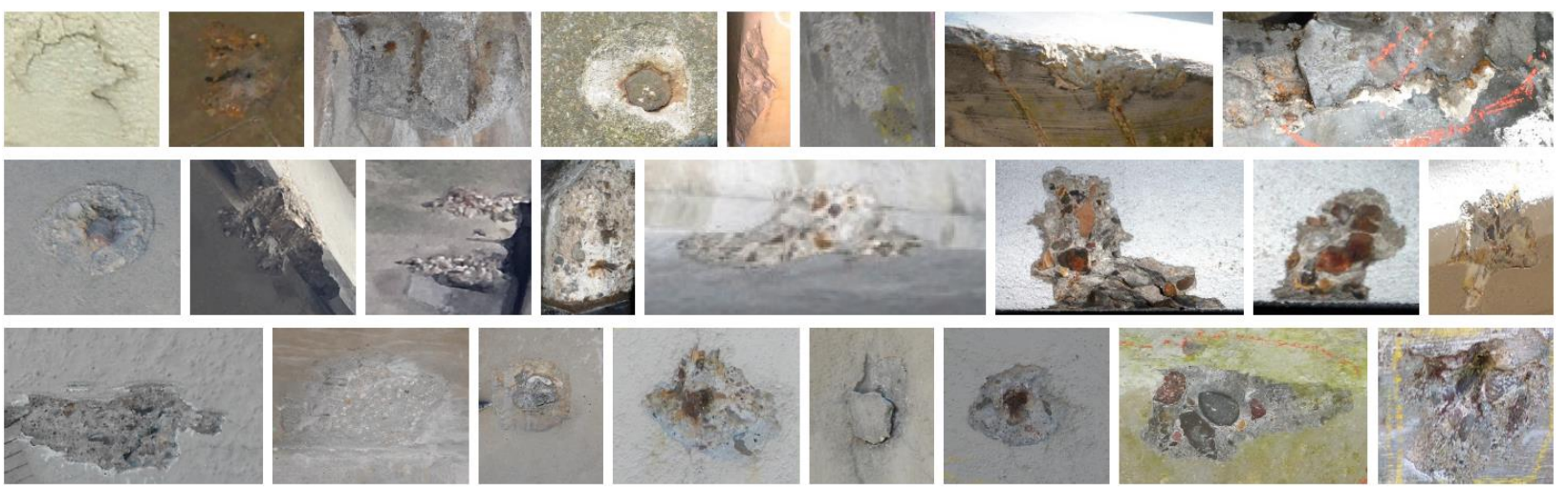

(h) Correctly classified images of class no exposed reinforcement (subset)


(i) Correctly classified images of class rust staining (subset) 

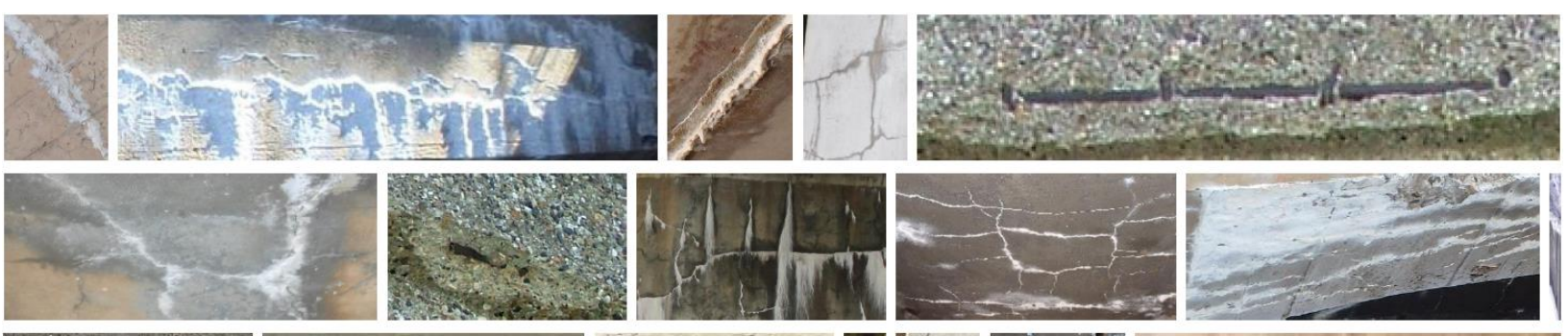

669
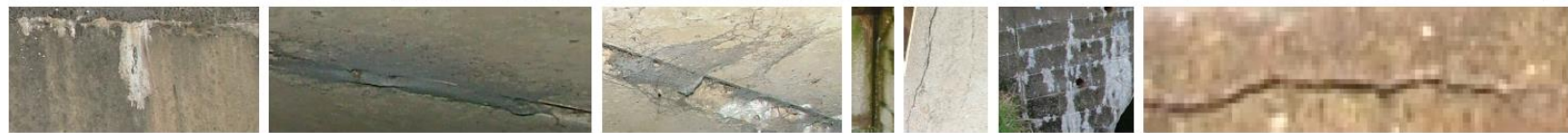

670

(j) Correctly classified images of class no rust staining (subset)

671

672

673 